Submitted to Nuclear Physics A

\title{
Thermal Evolution of Compact Stars
}

\author{
Christoph Schaab \\ Institut für theoretische Physik, Ludwig-Maximilians Universität München, \\ Theresienstr. 37, D-80333 München, Germany \\ email:schaab@gsm.sue.physik.uni-muenchen.de \\ Fridolin Weber \\ Institut für theoretische Physik, Ludwig-Maximilians Universität München, \\ Theresienstr. 37, D-80333 München, Germany \\ Manfred K. Weigel \\ Sektion Physik der Ludwig-Maximilians Universität München, Am Coulombwall 1, \\ D-85748 Garching, Germany \\ Norman K. Glendenning \\ Nuclear Science Division and Institute for Nuclear $\&$ Particle Astrophysics \\ Lawrence Berkeley National Laboratory, Berkeley, CA 94720, U.S.A. \\ PACS: 97.10.Cv, 97.60.Jd, 26.60+c, 12.38.Mh
}

A collection of modern, field-theoretical equations of state is applied to the investigation of cooling properties of compact stars. These comprise neutron stars as well as hypothetical strange matter stars, made up of absolutely stable 3-flavor strange quark matter. Various uncertainties in the behavior of matter at supernuclear densities, e.g., hyperonic degrees of freedom, behavior of coupling strengths in matter, pion and meson condensation, superfluidity, transition to quark matter, absolute stability of strange quark matter, and last but not least the many-body technique itself are tested against the body of observed cooling data.

Key words: dense matter, equation of state, neutron stars, strange stars, cooling 


\section{Introduction}

A forefront area of research, both experimental and theoretical, concerns the exploration of the properties of matter under extreme conditions of temperature and/or density and the determination of the equation of state (pressure versus density) associated with it. Its knowledge is of key importance for our understanding of the physics of the early universe, its evolution to the present day, compact stars, various astrophysical phenomena, and laboratory physics

(for an overview, see, for example, [1]). On the earth, relativistic heavy-ion colliders provide the only tool by means of which such matter can be created and its properties studied. On the other hand, however, it is well known that nature has created a large number of massive stellar objects, i.e., white dwarfs and neutron stars, which contain matter in one of the densest forms found in the universe. Neutron stars are associated with two classes of astrophysical objects - pulsars and compact X-ray sources. Matter in their cores possess densities ranging from a few times the density of normal nuclear matter to about an order of magnitude higher, depending on star mass. To the present day, about 600 pulsars are known, and the discovery rate of new ones is rather high. This is accompanied by an impressive growth rate of the body of observed pulsar data, like pulsar temperatures determined by the X-ray observatories Einstein, EXOSAT, and ROSAT [2 4].

In this paper, we shall apply a broad collection of modern, field-theoretical equations of state (EOS) to the study of the cooling behavior of both neutron stars and their strange counterparts - strange matter stars - which should exist if 3-flavor strange quark matter is more stable than confined hadronic matter. This collection of EOSs was derived under numerous model assumptions about the behavior of superdense stellar matter. To mention several are: the many-body technique used to determine the equation of state; the model for the nucleon-nucleon interaction; description of electrically charge neutral neutron star matter in terms of either only neutrons, neutrons and protons in generalized chemical equilibrium ( $\beta$ equilibrium) with electrons and muons, or nucleons, hyperons and more massive baryon states in $\beta$ equilibrium with leptons; behavior of the hyperon coupling strengths in matter, inclusion of meson $(\pi, K)$ condensation; treatment of the transition of confined hadronic matter into quark matter; and assumptions about the true ground state of strongly interacting matter (i.e., absolute stability of strange quark matter relative to baryon matter).

The paper is organized as follows. In section 2 we introduce the set of equations that govern the cooling behavior of massive stars. The collection of equations of state for neutron stars is discussed in section 3. The physics of strange stars and their associated EOS is explained in section 4 . The phenomenon of superfluidity and the various neutrino emission processes are outlined in 
sections 5 and 6 . Our results and conclusions are presented in sections 7 and 8, respectively. We summarize the paper in section 9 .

\section{Structure equations}

The structure of compact, massive stars is determined by Einstein's field equations,

$$
G^{\mu \nu}=8 \pi T^{\mu \nu}
$$

where $G^{\mu \nu}$ denotes the Einstein tensor and $T^{\mu \nu}$ is the energy-momentum tensor. For spherically symmetric, non-rotating stellar configurations the line element is given by (Schwarzschild metric)

$$
\mathrm{d} s^{2}=-\mathrm{e}^{2 \phi} \mathrm{d} t^{2}+\mathrm{e}^{2 \Lambda} \mathrm{d} r^{2}+r^{2} \mathrm{~d} \theta^{2}+r^{2} \sin ^{2} \theta \mathrm{d} \varphi^{2}
$$

where $\phi(r)$ and $\Lambda(r)$ denote the radially dependent metric functions.

Equations (1) and (2) combined with the law of energy-momentum conservation, $T_{; \nu}^{\mu \nu}=0$, lead to

$$
\begin{aligned}
\frac{\mathrm{d} r}{\mathrm{~d} N} & =\frac{1}{4 \pi r^{2} n \mathrm{e}^{\Lambda}}, \\
\frac{\mathrm{d} M}{\mathrm{~d} N} & =\frac{\rho}{n} \mathrm{e}^{-\Lambda}, \\
\frac{\mathrm{d} \phi}{\mathrm{d} N} & =\frac{G\left(\frac{4 \pi}{c^{2}} r^{3} P+M\right) \mathrm{e}^{\Lambda}}{4 \pi c^{2} r^{4} n}, \\
\frac{\mathrm{d} P}{\mathrm{~d} N} & =-\left(P+c^{2} \rho\right) \frac{\mathrm{d} \phi}{\mathrm{d} N},
\end{aligned}
$$

which describe the structure of static stars that are in general-relativistic hydrostatic equilibrium (Tolman-Oppenheimer-Volkoff equations, cf. [5, 6]). The metric function $\Lambda$ is determined by

$$
\mathrm{e}^{\Lambda}=\left(1-\frac{2 G M}{r c^{2}}\right)^{-\frac{1}{2}}
$$

The baryon number $N$ is introduced as an integration variable, since this is the only conserved quantity in relativistic field theory. $M(N)$ is the total mass 
within a sphere that contains $N$ baryons. Its surface area is equal to $4 \pi r^{2}(N)$. The quantity $P(N)$ denotes pressure as a function of baryon number, $\rho(P(N))$ is the total energy density, and $n(P(N))$ the baryon number density. Finally, we mention that the functions $\rho(P)$ and $n(P)$ are related to each other by the equation of state (EOS).

The corresponding boundary conditions are given by

$$
\begin{aligned}
r(N=0) & =0, \\
M(N=0) & =0, \\
\phi\left(N=N_{\mathrm{m}}\right) & =\frac{1}{2} \ln \left(1-\frac{2 G M\left(N=N_{\mathrm{m}}\right)}{r\left(N=N_{\mathrm{m}}\right) c^{2}}\right), \\
\rho\left(P\left(N=N_{\mathrm{m}}\right)\right) & =\rho_{\mathrm{m}} .
\end{aligned}
$$

The quantity $N_{\mathrm{m}}$ is defined by the relation

$$
M\left(N=N_{\mathrm{m}}\right)=M_{\mathrm{m}},
$$

where $M_{\mathrm{m}}$ denotes a given star mass.

The photosphere of the star is treated separately from the structure equations (3)-(6) for two reasons: Firstly, the equation of state of the photosphere depends much more sensitively on temperature than the core's equation of state, which can be treated at zero temperature; secondly, because of a scaling behavior (see, e.g., [7,8]) the structure equations of the photosphere need to be solved only once, for a properly chosen star mass. The interface between crust and photosphere is located at the density $\rho_{\mathrm{m}}$. We shall use the value given by Van Riper [8], $\rho_{\mathrm{m}}=10^{10} \mathrm{~g} \mathrm{~cm}^{-3}$.

Since the central star temperature drops down to less than $10^{9} \mathrm{~K}$ within a few minutes after birth [9], the effects of finite temperatures on the equation of state can be neglected to a very good approximation. Consequently, the four differential equations (3)-(6) do not depend on time and thus need to be solved only once, at the beginning of the numerical cooling simulation.

The thermal evolution is described by the partial differential equations of energy balance [6],

$$
\frac{\partial\left(L \mathrm{e}^{2 \phi}\right)}{\partial N}=-\frac{1}{n}\left(\epsilon_{\nu} \mathrm{e}^{2 \phi}+c_{\mathrm{v}} \frac{\partial\left(T \mathrm{e}^{\phi}\right)}{\partial t}\right)
$$

and thermal energy transport, 
Table 1

Input quantities for the cooling simulations performed in this paper

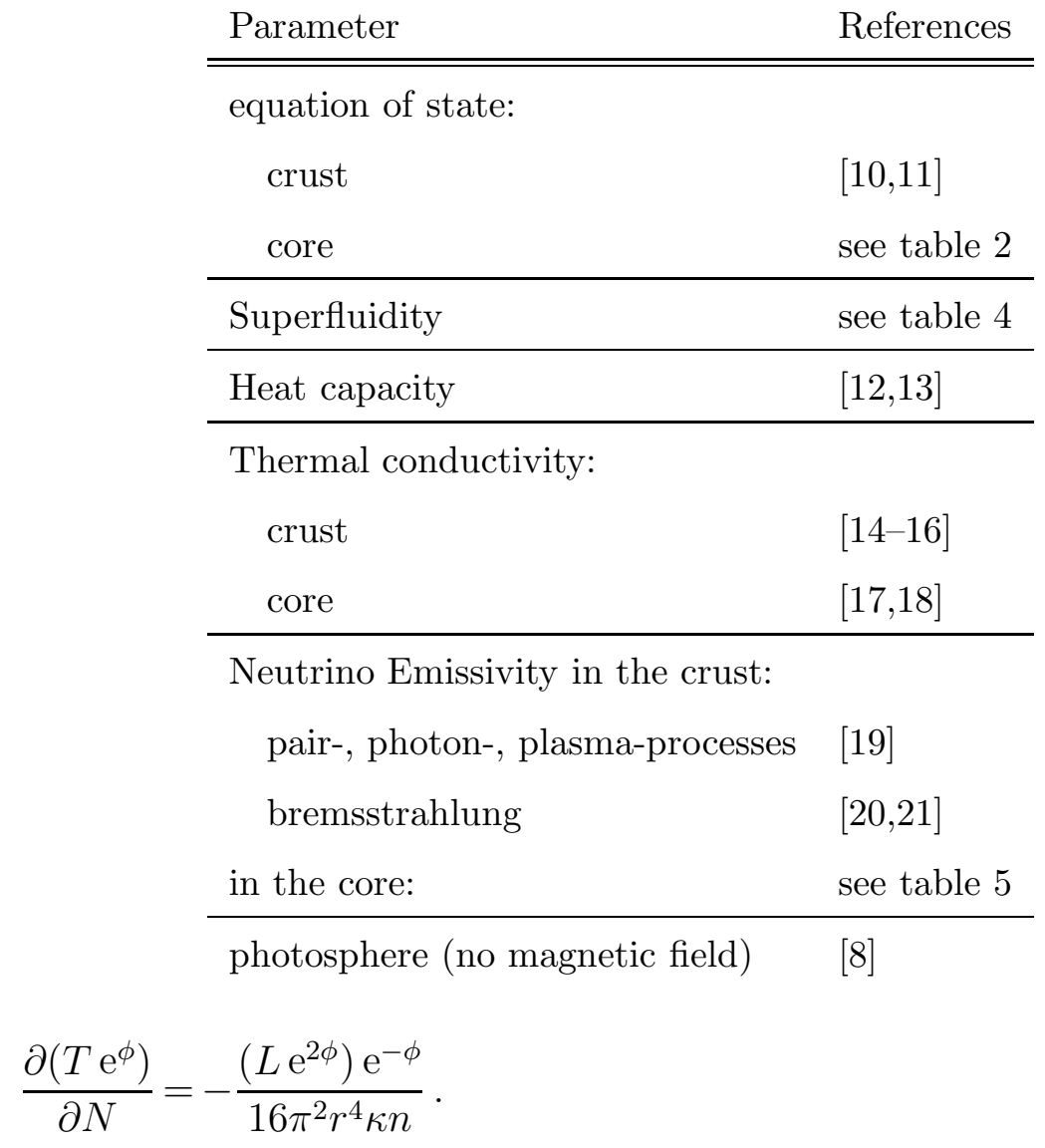

The microphysical input into Equations (13) and (14) are the neutrino emissivity per unit volume, $\epsilon_{\nu}(P, T)$, heat capacity per unit volume, $c_{\mathrm{v}}(P, T)$, and thermal conductivity, $\kappa(P, T)$. The boundary conditions for (13) and (14) read

$$
\begin{aligned}
L(N=0) & =0, \\
T\left(N=N_{\mathrm{m}}\right) & =T_{\mathrm{m}}\left(r_{\mathrm{m}}, L_{\mathrm{m}}, M_{\mathrm{m}}\right),
\end{aligned}
$$

where $T_{\mathrm{m}}\left(r_{\mathrm{m}}, L_{\mathrm{m}}, M_{\mathrm{m}}\right)$ is fixed by the properties of the photosphere at $r=r_{\mathrm{m}}$ [7,8]. The initial condition for the star's temperature can be assumed, without loss of generality, to be $T(r) \equiv 10^{11} \mathrm{~K}$, because the star's thermal evolution at times greater than say a few months does not depend on the exact initial temperature profile inside the star. Equations (13) and (14) were solved numerically by means of a Newton-Raphson-like algorithm. Table 1 summarizes the microphysical input quantities which enter the set of thermal structure equations, (3)-(6), (13) and (14). The input quantities will be discussed in detail in sections 3.6 . 


\section{Models for the equation of state}

\subsection{General remarks}

The equation of state (i.e., pressure and composition as function of energy density) of neutron star matter is the basic input quantity whose knowledge over a wide range of densities, ranging from the density of iron at the star's surface up to about 15 times the density of normal nuclear matter reached in the cores of the most massive star of a sequence, is necessary when solving the thermal structure equations. Since one is dealing with highly isospin-asymmetric, net-strangeness carrying matter in $\beta$-equilibrium whose properties cannot be explored in laboratory experiments, one is left with models for the equation of state which depend on theoretically motivated assumptions and/or speculations about the behavior of superdense matter. Sources of uncertainty concern, for instance, a competition between non-relativistic Schroedinger-based treatments versus relativistic field theoretical ones; moreover there exists even a considerable uncertainty with respect to the adequate many-body technique, which is to be introduced when solving the coupled equations of motion of many-baryon matter. Other items of uncertainty concern the baryon/meson composition of neutron star matter. This question has been treated in the literature at different levels of complexity. The simplest description approximates neutron star matter by pure neutron matter, which however is certainly not the ground state of neutron star matter. In fact neutron matter will quickly decay by means of the weak force into chemically equilibrated neutron star matter, whose fundamental constituents-besides neutrons-are protons, hyperons and possibly more massive baryons that become populated up to the highest densities reached in the cores of neutron stars. Finally we mention the possible transition of confined baryonic matter into quark matter, which, as demonstrated recently by one of us [22], may set in at a density less than twice normal nuclear matter density. Such densities are easily reached in neutron stars possessing canonical masses of $1.4 M_{\odot}$. Last but not least, there is the open problem concerning $K$ - or $\pi$-meson condensates in neutron stars.

The cross section of a neutron star can be split roughly into three distinct regimes. The first one is the star's outer crust, which consists of a lattice of atomic nuclei and a Fermi liquid of relativistic, degenerate electrons. The outer crust envelopes what is called the inner crust, which extends from neutron

drip density, $\rho=4.3 \times 10^{11} \mathrm{~g} \mathrm{~cm}^{-3}$, to a transition density of about $\rho_{\mathrm{tr}}=$ $1.7 \times 10^{14} \mathrm{~g} \mathrm{~cm}^{-3}$ [23]. Beyond $\rho_{\text {tr }}$ one enters the star's third regime, that is, its core where all atomic nuclei have dissolved into their constituents, protons and neutrons. Furthermore, as outlined just above, due to the high Fermi pressure the core will contain hyperons, eventually more massive baryon resonances, and possibly a gas of free up, down and strange quarks. Finally $\pi$ - and $K-$ 
meson condensates may be found there too. The equation of state of the outer and inner crust has been studied in several investigations and is rather well known. We shall adopt the models derived by Baym, Pethick and Sutherland [10] and Negele and Vautherin [11], respectively, for it. The models for the equation of state of the star's core will be discussed in detail in the next section. They fall into two categories, non-relativistic variational equations of state and the relativistic field theoretical ones.

\subsection{Non-relativistic variational approximation}

The non-relativistic models for the equation of state that are considered in our investigation use modern two-body interactions, $\mathbf{V}_{i j}$, whose parameters are adjusted to the properties of the deuteron and the free two-nucleon scattering problem. These two-body interactions are supplemented with phenomenological three-body interactions, $\mathbf{V}_{i j k}$. The additional free parameters are adjusted to the properties of nuclear matter and the properties of ${ }^{3} \mathrm{H}$ and ${ }^{4} \mathrm{He}$ [24]. The Hamiltonian's structure is given by

$$
\mathbf{H}=\sum_{i}\left(\frac{-\hbar^{2}}{2 m}\right) \nabla_{i}^{2}+\sum_{i<j} \mathbf{V}_{i j}+\sum_{i<j<k} \mathbf{V}_{i j k}
$$

The many-body method adopted to solve the many-body Schroedinger equation is the variational approach [24], where a variational trial wave-function, $\left|\Psi_{v}\right\rangle$, is constructed from a symmetrized product of two-body correlation operators, $F_{i j}$, which act on an unperturbed ground-state, i.e.,

$$
\left|\Psi_{v}>=\left[\mathbf{S} \prod_{i<j} \mathbf{F}_{i j}\right]\right| \Phi>
$$

The quantity $\mid \Phi>$ denotes the antisymmetrized Fermi-gas wave function. The correlation operator contains variational parameters which are varied to minimize the energy per baryon for a given baryon number density $n$ [24],

$$
E_{v}(n)=\min \left\{\frac{<\Psi_{v}|H| \Psi_{v}>}{<\Psi_{v} \mid \Psi_{v}>}\right\} .
$$

Equations (18) and (19) were solved in [24] simultaneously in combination with the equations for electric charge neutrality and $\beta$-equilibrium $\left(q_{B}\right.$ and $\mu^{B}$ denote electric charge and chemical potential of baryon $B$, respectively; 
$\mu^{\lambda}$ is the chemical potential of leptons, $\left.\lambda=e^{-}, \mu^{-}\right)$,

$$
\begin{gathered}
\sum_{B=p, n, \ldots} q_{B}\left(2 J_{B}+1\right) \frac{k_{F, B}^{3}}{6 \pi^{2}}-\sum_{L=e, \mu} \frac{k_{F, \lambda}^{3}}{3 \pi^{2}}=0, \\
\mu^{B}=\mu^{n}-q_{B} \mu^{e}, \quad \mu^{\mu}=\mu^{e},
\end{gathered}
$$

which relate the Fermi momenta, $k_{F, B}$ and $k_{F, \lambda}$ and chemical potentials of the various baryons and leptons with each other [25].

As representative models for the equation of state derived within this framework, we have selected a model derived by Wiringa, Fiks and Fabrocini [24, where the two-body interaction $\mathrm{UV}_{14}$ supplemented with the three-body interaction UVII, derived by the Urbana group, serve as an input. This equation of state takes only protons and neutrons in equilibrium with respect to the weak force into account.

\subsubsection{Relativistic, field theoretical approximations}

The structure of a lagrangian that accounts for the physics of neutron star matter is given by 25

$$
\mathcal{L}=\sum_{B} \mathcal{L}_{B}^{0}+\sum_{M} \mathcal{L}_{M}^{0}+\sum_{\lambda} \mathcal{L}_{\lambda}^{0}+\sum_{B, M} \mathcal{L}_{B, M}^{\mathrm{int}}+\mathcal{L}^{\left(\sigma^{4}\right)}
$$

where $B$ is summed over those baryons that become populated up to the highest densities reached in the cores of neutron stars, i.e., $B=\mathrm{n}, \mathrm{p}, \Sigma^{ \pm, 0}, \Lambda$, $\Xi^{0,-}, \Delta^{-}$. The quantity $M$ sums the mesons that are exchanged between them, $M=\sigma, \omega, \pi, \rho, \eta, \delta$, and $\phi$. The only contributing leptons are electrons and muons, hence $\lambda=\mathrm{e}^{-}, \mu^{-}$. The first three terms in (22) are the lagrangians of free particles. The term $\mathcal{L}_{B, M}^{\mathrm{int}}$ describes the interactions between the baryons, and $\mathcal{L}^{\left(\sigma^{4}\right)}$ represents self-interactions of $\sigma$ mesons.

By means of applying the Green's function technique to the equations of motion that follow from (22), one obtains the following coupled system of equations 25 28]. The first one is Dyson's equation which determines the two-point Green's functions, $g_{1}^{B}$,

$$
\left(\left(g_{1}^{0, B}\right)^{-1}(1,2)-\Sigma^{B}(1,2)\right) g_{1}^{B}\left(1,1^{\prime}\right)=\delta\left(1,1^{\prime}\right)
$$

The second equation determines the effective baryon-baryon scattering amplitude in matter, $T^{B B^{\prime}}$, 


$$
\begin{aligned}
\left\langle 12\left|T^{B B^{\prime}}\right| 1^{\prime} 2^{\prime}\right\rangle= & \left\langle 12\left|v^{B B^{\prime}}\right| 1^{\prime} 2^{\prime}-2^{\prime} 1^{\prime}\right\rangle \\
& +\left\langle 12\left|v^{B \bar{B}}\right| 34\right\rangle \Lambda^{\bar{B} \bar{B}^{\prime}}(34,56)\left\langle 56\left|T^{\bar{B}^{\prime} B^{\prime}}\right| 1^{\prime} 2^{\prime}\right\rangle,
\end{aligned}
$$

where $\Lambda$ stands for the Brueckner propagator [25,27 [30].

The system of equations is closed by the expression for the self energy of a baryon in matter, $\Sigma^{B}$,

$$
\Sigma^{B}(1,2)=-i\left\langle 13\left|T^{B B^{\prime}}\right| 42\right\rangle g_{1}(4,3) .
$$

We use the convention to sum or integrate over doubly occurring variables. The one-boson-exchange potential, $v$, sums the contributions arising from the various types of mesons,

$$
\left\langle 12\left|v^{B B^{\prime}}\right| 34\right\rangle=\sum_{M=\sigma, \omega, \pi, \rho, \ldots}\left\langle 12\left|v_{M}^{B B^{\prime}}\right| 34\right\rangle .
$$

To obtain a relativistic model for the equation of state of neutron star matter, equations (23) to (25) are to be solved self-consistently in combination with relations (20) and (21) for electric charge neutrality and chemical equilibrium. This has been done for different levels of approximation. With increasing level of complexity, these are the relativistic Hartree (RH), relativistic HartreeFock (RHF), and relativistic Brueckner-Hartree-Fock (RBHF) approximations [25.31. The latter scheme constitutes the most complex treatment that is presently manageable. It has been applied successfully to the composition of neutron star matter only recently. In contrast to RH and RHF, where the parameters of the theory, are to be adjusted to the properties of infinite nuclear matter, the RBHF method makes use of one-boson-exchange potentials whose parameters are adjusted to the properties of the deuteron and the relativistic nucleon-nucleon scattering problem. This leaves one with a so-called "parameter-free" treatment.

\subsection{Comparison of the equations of state}

An overview of the collection of equations of state used in this paper is given in table 2. This collection serves to study both the influence of competing many-body approximations as well as different models for the nuclear forces on the cooling behavior of neutron stars. Table 3 contains the corresponding nuclear matter properties. A graphical illustration of some of the equations of state is given in fig. 1.

With the exception of RBHF(B), the coupling constants of all other relativistic approximation schemes are chosen such that the properties of infinite nuclear 
Table 2

Dynamics and approximation schemes for equations of state derived for the cores of neutron stars

\begin{tabular}{|c|c|c|c|c|}
\hline EOS & Composition & Interaction & $\begin{array}{l}\text { Many body ap- } \\
\text { proach }\end{array}$ & Reference \\
\hline $\mathrm{UV}_{14}+\mathrm{UVII}$ & $\mathrm{p}, \mathrm{n}, \mathrm{e}^{-}, \mu^{-}$ & $\begin{array}{l}\text { 2-nuclei potential Urbana } V_{14} \text { and } \\
\text { 3-nuclei potential Urbana VII }\end{array}$ & $\begin{array}{l}\text { non-relativistic } \\
\text { variational } \\
\text { method }\end{array}$ & \\
\hline $\mathrm{HV}$ & $\begin{array}{l}\mathrm{p}, \mathrm{n}, \Lambda, \Sigma^{ \pm, 0}, \Xi^{0,-}, \mathrm{e}^{-} \\
\mu^{-}\end{array}$ & Exchange of $\sigma, \omega, \rho$ mesons & $\mathrm{RH}$ & 32 \\
\hline $\mathrm{RBHF}(\mathrm{B})+\mathrm{HFV}$ & $\begin{array}{l}\mathrm{p}, \mathrm{n}, \Lambda, \Sigma^{ \pm, 0}, \Xi^{0,-} \\
\Delta^{-}, \mathrm{e}^{-}, \mu^{-}\end{array}$ & Exchange of $\sigma, \omega, \pi, \rho \eta, \delta$ mesons & $\mathrm{RBHF}$ & 2728 \\
\hline $\mathrm{G}_{300}$ & $\begin{array}{l}\mathrm{p}, \mathrm{n}, \Lambda, \Sigma^{ \pm, 0}, \Xi^{0,-}, \mathrm{e}^{-}, \\
\mu^{-}\end{array}$ & Exchange of $\sigma, \omega, \rho$ mesons & $\mathrm{RH}$ & 33 \\
\hline $\mathrm{G}_{300}^{\pi}$ & $\begin{array}{l}\mathrm{p}, \mathrm{n}, \Lambda, \Sigma^{ \pm, 0}, \Xi^{0,-}, \mathrm{e}^{-} \\
\mu^{-}, \text {pion-condensate }\end{array}$ & Exchange of $\sigma, \omega, \rho$ mesons & $\mathrm{RH}$ & 33 \\
\hline $\mathrm{G}_{\mathrm{B} 180}^{\mathrm{K} 240}$ & $\begin{array}{l}\mathrm{p}, \mathrm{n}, \Lambda, \Sigma^{ \pm, 0}, \Xi^{0,-} \\
\mathrm{e}^{-}, \mu^{-}, \text {quark matter } \\
\text { phase }\end{array}$ & Exchange of $\sigma, \omega, \rho$ mesons & $\begin{array}{l}\text { RH, MIT bag } \\
\text { model }\end{array}$ & 34 \\
\hline $\mathrm{G}_{\mathrm{M} 78}^{\mathrm{K} 240}$ & 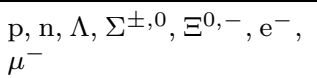 & Exchange of $\sigma, \omega, \rho$ mesons & $\mathrm{RH}$ & 34 \\
\hline
\end{tabular}

Abbreviations: $\mathrm{RH}=$ relativistic Hartree approximation, $\mathrm{RBHF}=$ relativistic

Brueckner-Hartree-Fock approximation.

Table 3

Properties of nuclear matter for the equations of state used in this work

\begin{tabular}{lccccc} 
EOS & $E / A[\mathrm{MeV}]$ & $n_{0}\left[\mathrm{fm}^{-3}\right]$ & $K[\mathrm{MeV}]$ & $M^{*}$ & $a_{\text {sy }}[\mathrm{MeV}]$ \\
\hline \hline $\begin{array}{l}\text { non-relativistic mod- } \\
\text { els }\end{array}$ & & & & & \\
$\mathrm{UV}_{14}+\mathrm{UVII}$ & -11.5 & 0.175 & 202 & 0.79 & 29.3 \\
\hline relativistic models & & & & & \\
$\mathrm{HV}$ & -15.98 & 0.145 & 285 & 0.77 & 36.8 \\
$\mathrm{RBHF}(\mathrm{B})$ & -15.73 & 0.172 & 249 & 0.73 & 34.3 \\
$\mathrm{G}_{300}$ & -16.3 & 0.153 & 300 & 0.78 & 32.5 \\
$\mathrm{G}_{300}^{\pi}$ & -16.3 & 0.153 & 300 & 0.78 & 32.5 \\
$\mathrm{G}_{\mathrm{B} 180}^{\mathrm{K} 240}$ & -16.3 & 0.153 & 240 & 0.78 & 32.5 \\
$\mathrm{G}_{\mathrm{M} 78}^{\mathrm{K} 240}$ & -16.3 & 0.153 & 240 & 0.78 & 32.5 \\
\hline
\end{tabular}

The entries are: saturation density, $n_{0}$; binding energy per baryon, $E / A$; compression modulus, $K$; effective mass in units of the nucleon mass, $M^{*}=$ $m^{*} / m$; asymmetry energy, $a_{\text {sy }}$. 


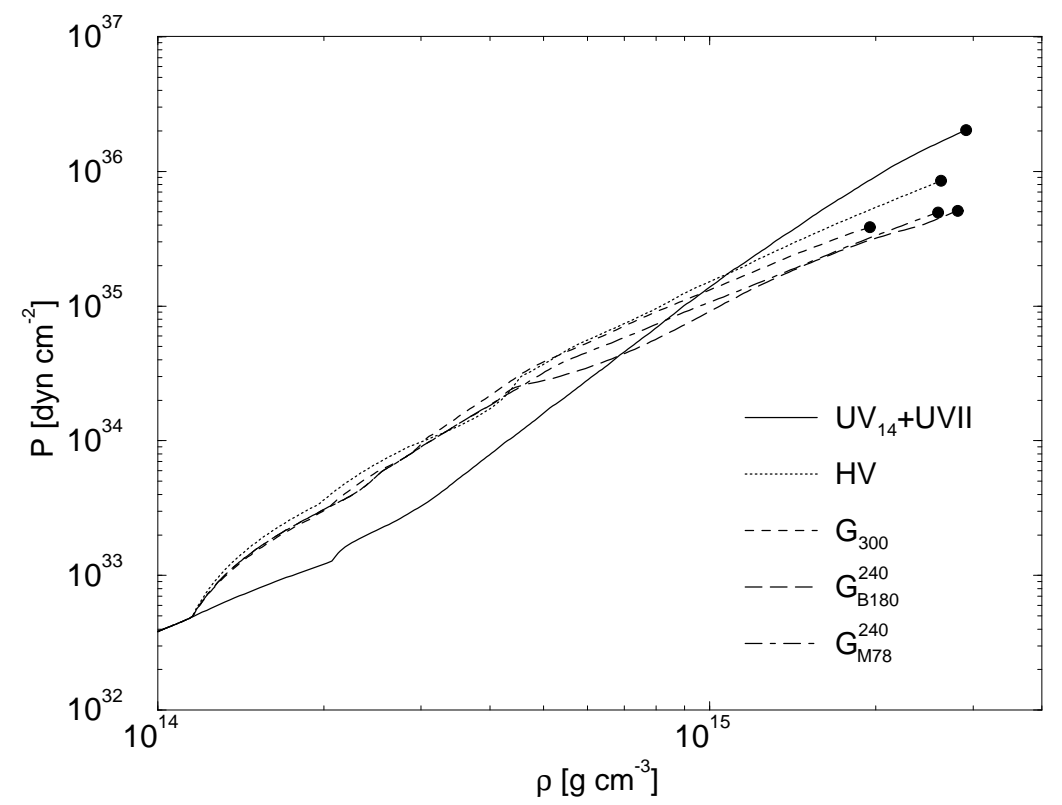

Fig. 1. Pressure-density relation for several EOSs. The dots mark the highest central mass density reached in the most massive neutron star of each sequence. Neutron stars with still higher central densities are unstable against compressional modes.

matter $\left(n_{0} \approx(0.15-0.16) \mathrm{fm}^{-3}, E / A \approx-16 \mathrm{MeV}, m^{*} \approx 0.78, K=200-300\right.$ $\left.\mathrm{MeV}, a_{\text {sy }} \approx 32 \mathrm{MeV}\right)$ can be reproduced. For RBHF(B), Brockmann's oneboson-exchange potential (version 'B') was used as an input, which, as pointed out above, leads to a so-called parameter-free treatment.

The ratio of hyperon to nucleon couplings to the meson fields,

$$
x_{\sigma}=g_{H \sigma} / g_{N \sigma}, \quad x_{\omega}=g_{H \omega} / g_{N \omega}, \quad x_{\rho}=g_{H \rho} / g_{N \rho},
$$

are not defined by the ground-state properties of normal nuclear matter and so must be chosen according to other considerations [35]. The binding energy of the $\Lambda$, for example, serves to strictly correlate the values of $x_{\sigma}$ and $x_{\omega}$, but leaves a continuous pairwise ambiguity which hypernuclear levels and neutron-star masses are able to resolve [35. In this paper we choose for the hyperon-to-nucleon scalar coupling $x_{\sigma}$ a minimum allowable value of $x_{\sigma} \sim 0.5$ found from the lower bound on the maximum neutron-star mass that is also consistent with the $\Lambda$ binding in nuclear matter. The corresponding value of $x_{\omega}$ amounts $\sim 0.52$.

The possible transition of confined hadronic matter into quark matter at high densities is contained in the equation of state labeled $\mathrm{G}_{\mathrm{B} 180}^{\mathrm{K} 240}$ [34. The transition was determined for a bag constant of $B^{1 / 4}=180 \mathrm{MeV}$ which places the energy per baryon of strange quark matter at $1100 \mathrm{MeV}$, well above the energy per nucleon in infinite nuclear matter as well as the most stable nucleus, ${ }^{56} \mathrm{Fe}$ $(E / A \approx 930 \mathrm{MeV})$. (The possibility of absolutely stable strange quark matter will be considered in sect. $甘$.) Most interestingly, this model predicts a transi- 


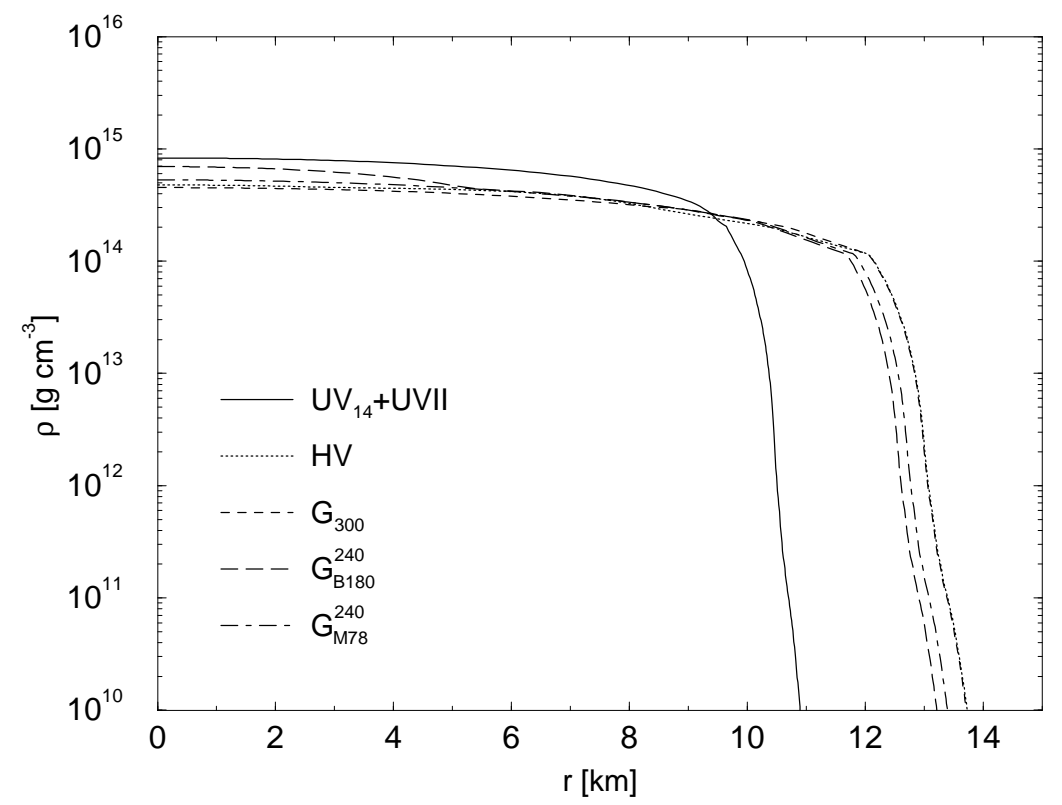

Fig. 2. Density profiles of neutron star with mass $1.0 M_{\odot}$ constructed for a sample of different EOSs.

tion to quark matter at a density as low as $1.6 n_{0}$. The pure quark-matter phase is reached at a density of about $10 n_{0}$, which is close to the central density of the heaviest and thus most compact star constructed from such an equation of state. We stress that these density thresholds are rather different from those obtained by numerous other authors, who performed work on this topic prior to Glendenning's paper. The reason for this lies in the seemingly innocuous assumption of treating neutron star matter as a simple body, that is, as matter that is characterized by only one conserved charge. This is not true for neutron star matter which possesses two conserved charges, baryonic as well as electric. Correspondingly, there are two chemical potentials rather than one, and the transition of baryon matter to quark matter is to be determined in the three-dimensional space spanned by pressure and the chemical potentials of the electrons and neutrons. The only existing investigations which accounts for this properly are those of Glendenning [22, 34, 36].

The stiffness of the equation of state depends strongly on the internal degrees of freedom. Generally, the more degrees of freedom accessible to a system, the softer the equation of state. A softer equation of state, in turn, leads to neutron stars that are less massive but more dense than those obtained for stiffer equations of state. This is illustrated in figs. 2 to 1 , where the density profiles of neutron stars constructed for different equations of state are shown. The stiffness of these equations of state can be inferred from fig. 且. 


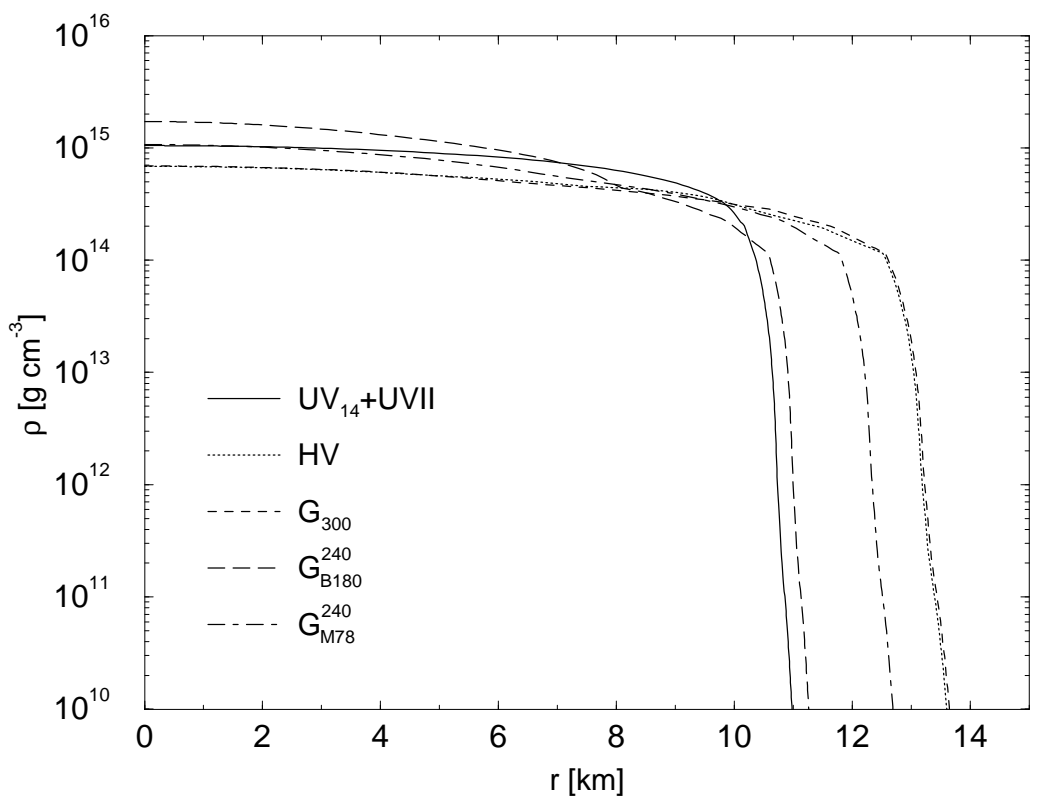

Fig. 3. Same as fig. 2 but for a neutron star with mass $1.4 M_{\odot}$.

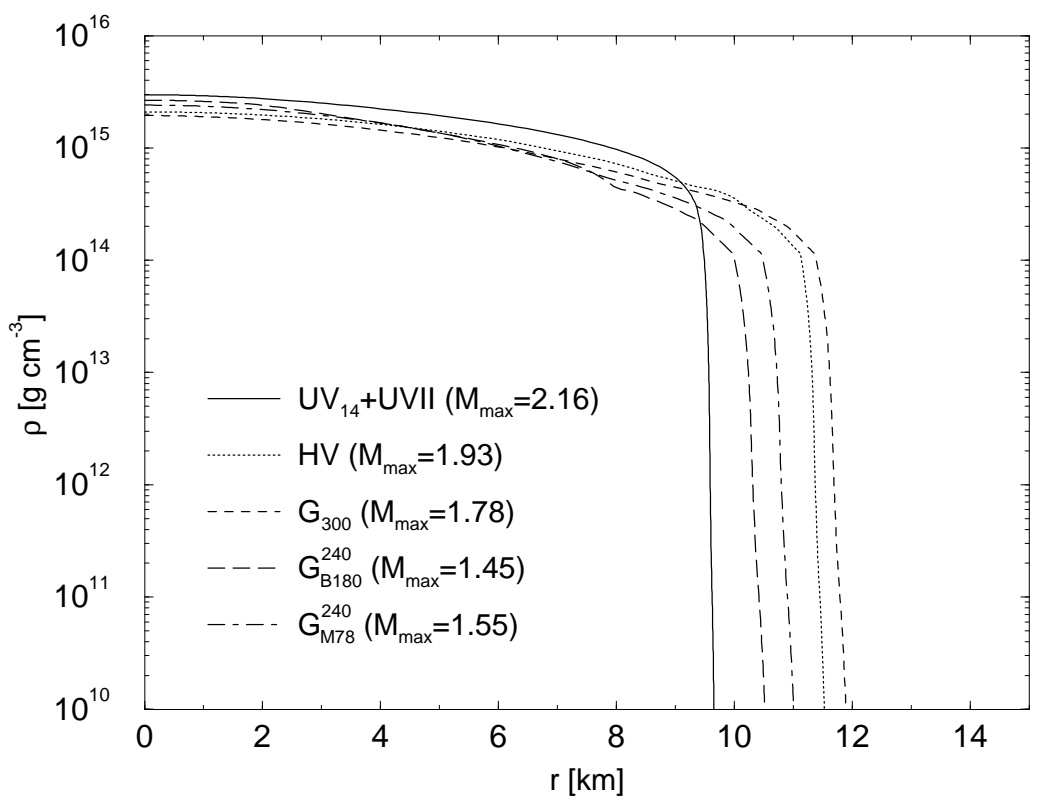

Fig. 4. Same as fig. 2 but for the heaviest neutron star of each sequence.

\section{Strange Stars}

The hypothesis that strange quark matter may be the absolute ground state of the strong interaction (not ${ }^{56} \mathrm{Fe}$ ) has been raised independently by Bodmer [37 and Witten [38. If the hypothesis is true, then separate classes of compact stars could exist, which range from dense strange stars to strange dwarfs to strange planets 39 41. They form distinct and disconnected branches of compact stars and are not part of the continuum of equilibrium configurations that include ordinary white dwarfs and neutron stars. In principle both strange and neutron stars could exist. However if strange stars exist, the galaxy is likely 
to be contaminated by strange quark nuggets which would convert all neutron stars that they come into contact with to strange stars 42 44. This would imply that the objects known to astronomers as pulsars are probably rotating strange matter stars and not neutron matter stars, as is usually assumed. Unfortunately, lattice QCD calculations will not be accurate enough in the foreseeable future to give a definitive prediction as to the absolute stability of strange matter so that even to the present day there is not sound scientific basis on which one can either confirm or reject the hypothesis, so that it remains a serious possibility of fundamental significance for compact stars plus various other physical phenomena [45]. Below we will explore the cooling behavior of strange stars and compare it with the one of their non-strange counterparts, i.e., ordinary neutron stars. This enables one to test the possible existence of strange stars and thus draw definitive conclusions about the true ground-state of strongly interacting matter.

As pointed out by Alcock, Farhi, and Olinto [46], a strange star can carry a solid nuclear crust whose density at its base is strictly limited by neutron drip. This is made possible by the displacement of electron at the surface of strange matter, which leads to a strong electric dipole layer there. It is sufficiently strong to hold open a gap between ordinary atomic (crust) matter and the quark-matter surface, which prevents a conversion of ordinary atomic matter into the assumed lower-lying ground state of strange matter. Obviously, free neutrons, being electrically charge neutral, cannot exist in the crust, because they do not feel the Coulomb barrier and thus would gravitate toward the strange-quark matter core, where they are converted by hypothesis into strange matter. Consequently, the density at the base of the crust (inner crust density) will always be smaller than neutron drip density. The situation is illustrated in fig. 5 where the equation of state of a strange star with crust is shown.

The strange-star models presented in the following are constructed for an equation of state of strange matter derived by Farhi and Jaffe [47]. The value chosen for the bag constant and strange quark mass are $B^{1 / 4}=145 \mathrm{MeV}$ and $m_{\mathrm{s}}=150 \mathrm{MeV}$, respectively. These choices place the energy per baryon of strange quark matter at $850 \mathrm{MeV}$, which corresponds to strongly self-bound strange matter.

\section{Superfluidity}

The effective nucleon-nucleon interaction contains a rather strong repulsive, short-range part and a weaker, attractive long-range part. Hence, depending on the average distance of the particles, nuclear matter may become super-

fluid (see [48] for a recent review). Neutrons, for instance, are believed to 


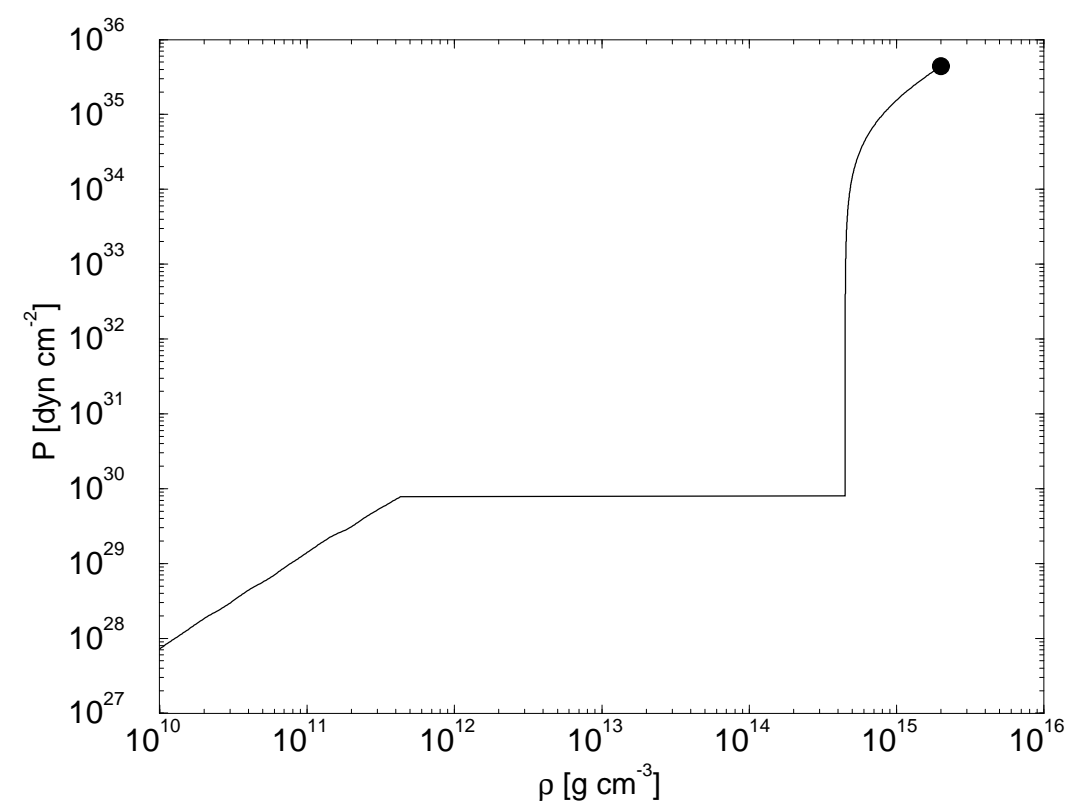

Fig. 5. Pressure-density relation for strange stars with crust. The bag constant is $B^{1 / 4}=145 \mathrm{MeV}$, the mass of the strange quark is $m_{s}=150 \mathrm{MeV}$. The solid dot marks the central density reached in the heaviest stable strange star. Stars with still higher central densities are unstable against compressional modes.

form ${ }^{1} \mathrm{~S}_{0}$ superfluid pairs in the density range between neutron drip and and the saturation density of normal nuclear matter (see below). At supernuclear densities, i.e., in the core region of neutron stars, the repulsive interaction becomes dominant and the ${ }^{1} \mathrm{~S}_{0}$ superfluidity gap closes. However, the tensor and spin-orbit interaction become attractive at such densities and the formation of ${ }^{3} \mathrm{P}_{2}$-neutron pairs becomes possible. Finally, the proton concentration in the core region may reach a partial density simlar to that of the pair-condensed neutron gas at subnuclear densities, enabling the protons to form superfluid ${ }^{1} \mathrm{~S}_{0}$ pairs. Since the protons are charged, the proton superfluid is a superconductor. The different superfluid regimes can be summarized as follows [49 51]:

(i) $7 \times 10^{11} \mathrm{~g} \mathrm{~cm}^{-3}$ to $2 \times 10^{14} \mathrm{~g} \mathrm{~cm}^{-3}$ : neutrons in ${ }^{1} \mathrm{~S}_{0}$-pair state,

(ii) $2 \times 10^{14} \mathrm{~g} \mathrm{~cm}^{-3}$ to $4 \times 10^{14} \mathrm{~g} \mathrm{~cm}^{-3}$ : protons in ${ }^{1} \mathrm{~S}_{0}$-pair state, (iii) $2 \times 10^{14} \mathrm{~g} \mathrm{~cm}^{-3}$ to $5 \times 10^{16} \mathrm{~g} \mathrm{~cm}^{-3}$ : neutrons in ${ }^{3} \mathrm{P}_{2}$-pair state.

One has to keep in mind that these values depend on the composition of matter. The above values are computed for the equation of state labeled HV. A further possibility not mentioned so far are ${ }^{3} \mathrm{D}_{2}$ neutron-proton pairs. Finally, it is possible that hyperons and quarks form superfluid states too [52,53. Until now, however, no detailed calculations on this topic exist and one is thus left with parameter studies (cf. section 7.7).

If protons and/or neutrons become superfluid, the neutrino emissivity, thermal conductivity, and heat capacity are reduced by an exponential factor of the 
Table 4

Gap energies and critical temperatures of superfluid states in neutron star matter

\begin{tabular}{cccc} 
& Neutron ${ }^{1} \mathrm{~S}_{0}$ & Neutron ${ }^{3} \mathrm{P}_{2}$ & Proton ${ }^{1} \mathrm{~S}_{0}$ \\
\hline \hline Reference & 49 & 50 & 51 \\
\hline$\Delta_{\mathrm{sf}}^{\max }[\mathrm{MeV}]$ & 1.13 & 0.62 & 0.25 \\
$T_{c}^{\max }\left[10^{9} \mathrm{~K}\right]$ & 7.4 & 0.8 & 1.6 \\
\hline from $\rho\left[\mathrm{g} \mathrm{cm}^{-3}\right]$ & $7 \times 10^{11}$ & $2 \times 10^{14}$ & $2 \times 10^{14}$ \\
to $\rho\left[\mathrm{g} \mathrm{cm}^{-3}\right]$ & $2 \times 10^{14}$ & $5 \times 10^{16}$ & $4 \times 10^{14}$
\end{tabular}

The density ranges are calculated for the HV equation of state. $\Delta_{\mathrm{sf}}^{\max }=$ $1.13 \mathrm{MeV}$ is obtained for the ${ }^{1} \mathrm{~S}_{0}$ superfluid gap as estimated by Ainsworth et al. Using the gaps estimated in the more recent paper by Wambach et al. 49] leads to $\Delta_{\mathrm{sf}}^{\max }=1.0 \mathrm{MeV}$, which influences the cooling of neutron stars only insignificantly.

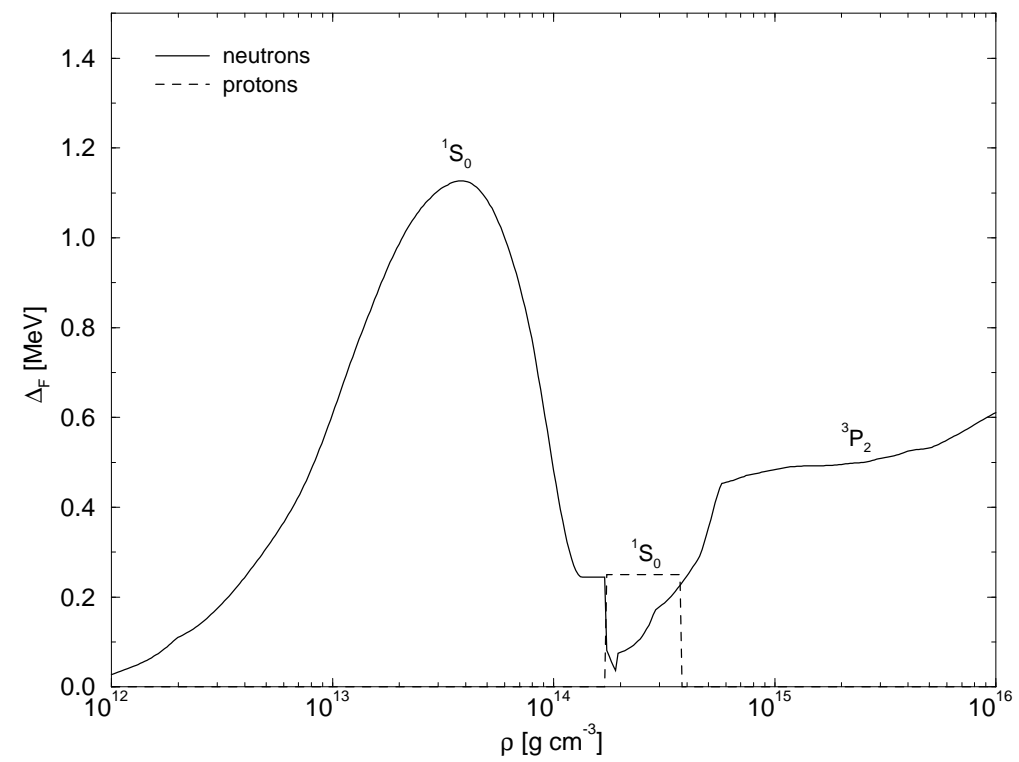

Fig. 6. Energy gap versus mass density computed for the HV EOS.

form円 $\exp \left(-\Delta_{\mathrm{sf}} / k_{\mathrm{B}} T\right)$, where $\Delta_{\mathrm{sf}}$ is the gap energy [17,56]. Unfortunately, the gap energies are not very well known because of uncertainties in the effective baryon masses and polarization effects [57]. We will take the gap energies for protons from [51], and those for the ${ }^{1} \mathrm{~S}_{0}$ and ${ }^{3} \mathrm{P}_{2}$ states of neutrons from [49] and [50, respectively. An overview of the gap energies and critical temperatures is put together in table 4 . As an example, we show in fig. 6 the density dependence of $\Delta_{\text {sf }}$ for one of our model equations of state. A qualitatively similar behavior is obtained for the other models.

$\overline{1 \text { For the }}{ }^{3} \mathrm{P}_{2}$ state this is rigorously true only for isotropic matter 53.55$]$. 
Table 5

Neutrino processes studied in this work, their associated neutrino emissivities, $\epsilon_{\nu}$, and the critical threshold densities, $n_{\text {crit }}$, below which these processes are forbidden

\begin{tabular}{cccc} 
Process & $\epsilon_{\nu}\left[\mathrm{erg} \mathrm{s}^{-1}\right]$ & $n_{\text {crit }}\left[\mathrm{fm}^{-3}\right]$ & Reference \\
\hline \hline Nucleon bremsstrahlung & $10^{19} \times T_{9}^{8}$ & & \\
modified Urca & $10^{21} \times T_{9}^{8}$ & \\
direct Urca & $10^{27} \times T_{9}^{6}$ & $0.25-0.5$ \\
Pion-condensate & $10^{26} \times T_{9}^{6}$ & 0.21 & 6.58 \\
Kaon-condensate & $10^{25} \times T_{9}^{6}$ & 0.71 \\
Quark $\beta$-decay & $10^{24} \times T_{9}^{6}$ & 0.29 & 60
\end{tabular}

The first two processes are slow ones. They are possible at all densities, for which bulk nuclear matter exisits. The critical density for the direct Urca process depends on the equation of state, which leads to a range of threshold densities.

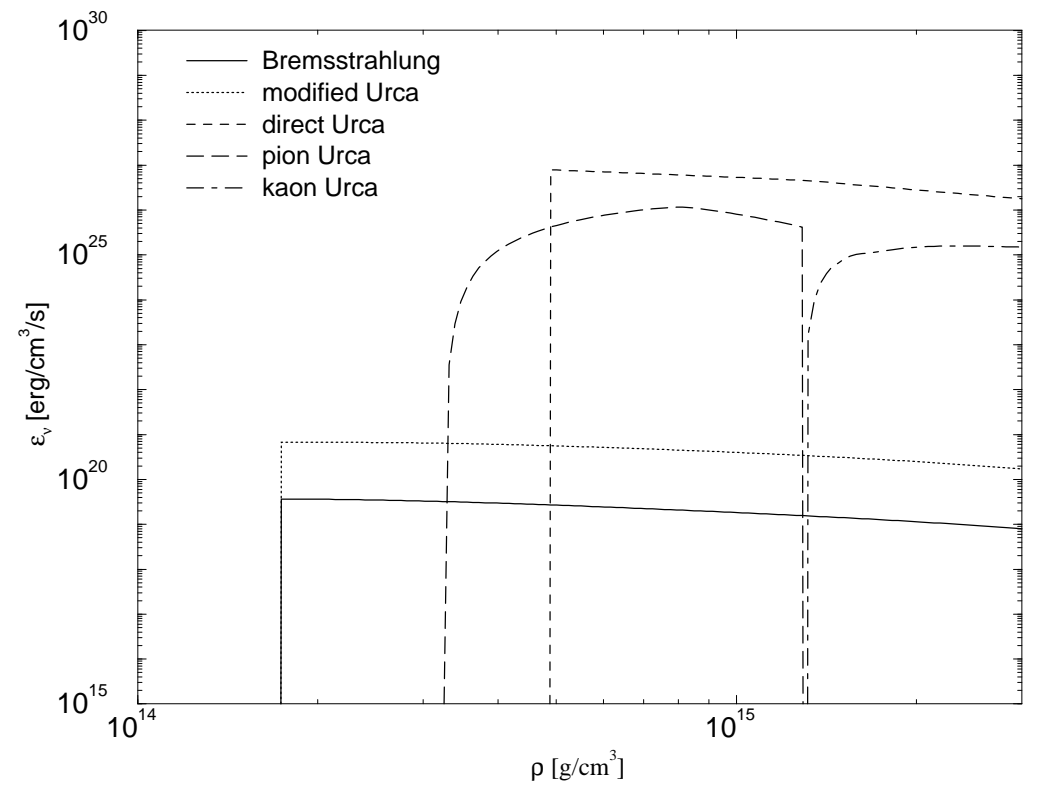

Fig. 7. Neutrino emissivities of different neutrino-emitting processes as a function of energy density for $T=10^{9} \mathrm{~K}$. The particle composition is taken from the $\mathrm{G}_{300}^{\pi}$ EOS.

\section{$6 \quad$ Neutrino emissivities}

The neutrino emission processes can be divided into slow and fast ones. The main feature, which distinguishes fast from slow processes, is the existence of a certain threshold density for the fast ones, below which they cannot take place. An overview of all processes which we shall take into account is given in table 5. The associated neutrino emissivities are shown in fig. 7 . 


\subsection{Neutrino pair Bremsstrahlung}

The most important neutrino process in the crust is neutrino-antineutrino pair production by electron bremsstrahlung. In early estimates it was found that the contribution of this process to cooling is competitive with standard cooling via the modified Urca process. However, recent investigations have shown that the mass of the ionic crust is reduced by a factor of two to three if one considers the recently established value $\rho_{\mathrm{tr}} \approx 1.7 \times 10^{14} \mathrm{~g} \mathrm{~cm}^{-3}$ for the transition density between crust and core [23], instead of the older value $\rho_{\text {tr }} \approx 2.8 \times 10^{14} \mathrm{~g} \mathrm{~cm}^{-3}$ [10]. Additionally, considerations by Pethick and Thorsson [21] have pointed out that the standard perturbative treatment of determining $\epsilon_{\nu}$ is not sufficiently accurate below a temperature of $10^{10} \mathrm{~K}$. Furthermore taking the electronic band structure into account can reduce the energy emission rates exponentially for temperatures below $10^{10} \mathrm{~K}$. We include these corrections to the emissivities derived by Itoh et al. in [64, 65]. Additionally to the above process, pair, photon and plasmon processes may also be important in the low density regime. Their effects have been estimated too by Itoh et al. in [19], and will be used in our calculations.

Neutrino-antineutrino pair bremsstrahlung production can also occur in the core according to

$$
\begin{gathered}
\mathrm{n}+\mathrm{n} \rightarrow \mathrm{n}+\mathrm{n}+\nu+\bar{\nu}, \\
\mathrm{n}+\mathrm{p} \rightarrow \mathrm{n}+\mathrm{p}+\nu+\bar{\nu}, \\
\mathrm{e}^{-}+\mathrm{p} \rightarrow \mathrm{e}^{-}+\mathrm{p}+\nu+\bar{\nu}
\end{gathered}
$$

We note that the emissivity of the last bremsstrahlung process, Equation (30), becomes very important if the neutrons become superfluid. In this case this process dominates all other processes. For the processes (28) to (30) we use the emissivities as calculated by Friman and Maxwell [56,58].

\subsection{Modified Urca process}

The beta decay and electron capture processes,

$$
\mathrm{n} \rightarrow \mathrm{p}+\mathrm{e}^{-}+\bar{\nu}_{\mathrm{e}} \quad \text { and } \quad \mathrm{p}+\mathrm{e}^{-} \rightarrow \mathrm{n}+\nu_{\mathrm{e}}
$$

also known as the direct Urca processes, were not included in cooling calculations [66 70] until recently, since energy and momentum conservation can only be fulfilled if the proton fraction exceeds a critical value (cf. section 6.3).

Most interestingly, many of the older, non-relativistic models for the equations 
of state of neutron star matter indeed do not lead to sufficiently high enough proton fractions. Consequently, the so-called modified Urca process, which is characterized by the occurrence of a bystander particle which is necessary to conserve both energy and momentum of the baryons that are being scattered, i.e.,

$$
\mathrm{n}+\mathrm{n} \rightarrow \mathrm{n}+\mathrm{p}+\mathrm{e}^{-}+\bar{\nu}_{\mathrm{e}} \quad \text { and } \quad \mathrm{n}+\mathrm{p}+\mathrm{e}^{-} \rightarrow \mathrm{n}+\mathrm{n}+\nu_{\mathrm{e}} .
$$

were considered to be the dominant cooling mechanism of neutron stars. The emission rates associated with processes (32) were calculated by Friman and Maxwell [58]. There the exchanged $\pi$ meson is treated as a free particle. Such an approximation neglects the influence of the medium on the propagating pion. Furthermore, the exchanged pions undergo weak transitions (so-called exchange current effect). An estimate of the influence of such effects, performed by Voskresenskii and Senatorov [71,72, shows that the neutrino emissivities may increase by up to several orders of magnitude. The implications of these effects of cooling will be studied in a different investigation [73].

\subsection{Direct Urca process}

As already mentioned, the direct Urca process (31) is only possible if the proton fraction exceeds a certain critical value [59,74]. If the star is assumed to be made up of only neutrons, protons and electrons, the critical proton fraction amounts about $11 \%$. In one includes hyperons and muons too, the critical value increases slightly to $13 \%$. In contrast to older, non-relativistic equations of state which predict proton fractions that lie below the critical values, modern non-relativistic as well as relativistic equations of state contain sufficiently large enough proton fractions such that the direct Urca process becomes possible. The proton fraction depends crucially on the asymmetry energy which unfortunately is not known at higher densities. From our study we find that the proton fraction is generally larger for the relativistic equations of state than for the non-relativistic ones. This trend can be seen in fig. 8 , where the proton fractions are shown for several equations of state of our collection. The critical energy densities beyond which the direct Urca becomes possible, are indicated. Because of the different asymmetry behaviors of these equations of state, the critical densities vary considerably from one equation of state to another. This, in turn, links the onset of the direct Urca process to the condition of a sufficiently large enough star mass. For the emissivities of the direct Urca process we shall use those calculated by Lattimer et al. [59]. The emissivity of the direct process exceeds the one of the modified process by up to ten orders of magnitude, at low temperatures of $T \approx 10^{7} \mathrm{~K}$.

So far we have considered the direct Urca process in neutron star matter made 


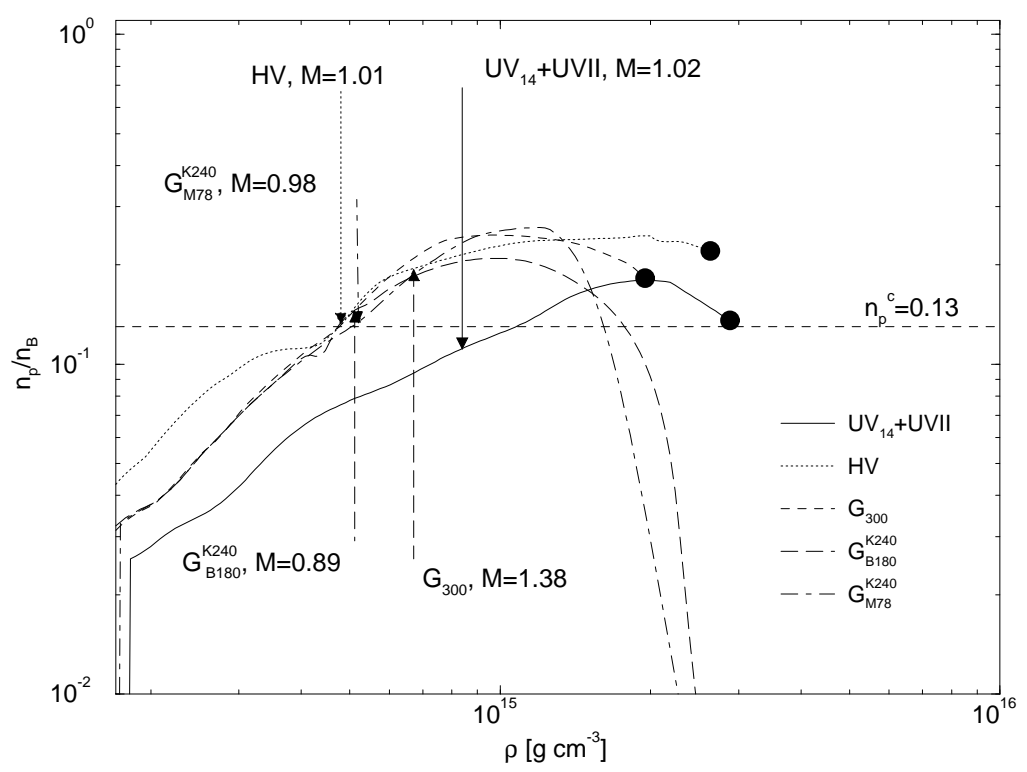

Fig. 8. Proton fraction in neutron star matter as a function of energy density for different equations of state. The approximate critical proton fraction $n_{\mathrm{p}} / n_{\mathrm{B}}=0.13$ for the direct Urca process is marked by the horizontal line. The exact critical energy densities together with the corresponding star (masses in units of $M_{\odot}$ ) are marked by arrows.

up of protons and neutrons only. Strange baryons and $\Delta$ isobars, however, which may exist in such matter rather abundantly too, also produce neutrinos via direct Urca processes of the following type,

$$
\Sigma^{-} \rightarrow \Lambda+\mathrm{e}^{-}+\bar{\nu}_{\mathrm{e}} \quad \text { and } \quad \Lambda+\mathrm{e}^{-} \rightarrow \Sigma^{-}+\nu_{\mathrm{e}}
$$

Such processes have been studied first by Prakash et al. [75, and the implications for cooling of neutron stars were explored in [76]. We have included the above processes in cooling simulations elsewhere [77. It was found that the cooling behavior due to direct Urca among the nucleons only dominates over the energy loss associated with processes the (33). Or, in quantitative terms, the cooling curves obtained by restriction to the direct Urca process among non-superfluid nucleons (section 7.4) remain practically unchanged.

\subsection{Quasi-particle processes in boson-condensed matter}

In the charged pion $\left(\pi^{\mathrm{c}}-\right)$ condensed phase, the quasi-particle Urca processes

$$
\eta(\pi) \rightarrow \eta(\pi)+\mathrm{e}^{-}+\bar{\nu}_{\mathrm{e}} \quad \text { and } \quad \eta(\pi)+\mathrm{e}^{-} \rightarrow \eta(\pi)+\nu_{\mathrm{e}}
$$


are possible. The quasi-particle state $\eta$ is given by the superposition of proton and neutron states,

$$
\left|\eta_{ \pm}\right\rangle=\cos \theta|\mathrm{n}\rangle \mp i \sin \theta|\mathrm{p}\rangle
$$

with the mixing angle $\theta$. The emissivity for this process was calculated by Maxwell et al. 60].

In recent years, some authors made a strong case for the condensation of kaons in the cores of neutron stars (see, for instance, [78 80]). The critical density beyond which this process may take place is uncertain. Estimates lie in the range of $3-5 n_{0}[61,81,82]$. The emissivities of the quasi-particle direct Urca processes,

$$
\mathrm{n}(\mathrm{K}) \rightarrow \mathrm{p}(\mathrm{K})+\mathrm{e}^{-}+\bar{\nu}_{\mathrm{e}} \quad \text { and } \quad \mathrm{p}(\mathrm{K})+\mathrm{e}^{-} \rightarrow \mathrm{n}(\mathrm{K})+\nu_{\mathrm{e}}
$$

are reduced, relative to the uncondensed case, by a factor of $\cos ^{2}(\theta / 2)$ [62. Additionally to (36) there are two more processes,

$$
\begin{array}{lll}
\mathrm{n}(\mathrm{K}) \rightarrow \mathrm{n}(\mathrm{K})+\mathrm{e}^{-}+\bar{\nu}_{\mathrm{e}} & \text { and } & \mathrm{n}(\mathrm{K})+\mathrm{e}^{-} \rightarrow \mathrm{n}(\mathrm{K})+\nu_{\mathrm{e}} \\
\mathrm{p}(\mathrm{K}) \rightarrow \mathrm{p}(\mathrm{K})+\mathrm{e}^{-}+\bar{\nu}_{\mathrm{e}} & \text { and } & \mathrm{p}(\mathrm{K})+\mathrm{e}^{-} \rightarrow \mathrm{p}(\mathrm{K})+\nu_{\mathrm{e}}
\end{array}
$$

whose emissivities were calculated by Thorsson et al. 62. The density dependence of the mixing angle $\theta$ and the value of the critical density, $n_{\mathrm{c}}=4.18 n_{0}$ are taken from 61.

\subsection{Quark processes}

The possible existence of quark matter in the cores of neutron stars has already been outlined in sect. 3.3. The latest calculations on this topic predict an onset of the transition of confined hadronic matter into quark matter at $n \approx 1.6 n_{0}$. (This is not to be confused with the possibility of absolutely stable strange matter, to which we will return in sect. (1).

The following weak processes are important in quark matter consisting of up, down and strange quarks,

$$
\begin{array}{lll}
\mathrm{d} \rightarrow \mathrm{u}+\mathrm{e}^{-}+\bar{\nu}_{\mathrm{e}} & \text { and } & \mathrm{u}+\mathrm{e}^{-} \rightarrow \mathrm{d}+\nu_{\mathrm{e}} \\
\mathrm{s} \rightarrow \mathrm{u}+\mathrm{e}^{-}+\bar{\nu}_{\mathrm{e}} & \text { and } & \mathrm{u}+\mathrm{e}^{-} \rightarrow \mathrm{s}+\nu_{\mathrm{e}}
\end{array}
$$

We shall use the neutrino emission rates given by Duncan, Shapiro, and Wasserman 63 to simulate the cooling history of neutron stars containing a 


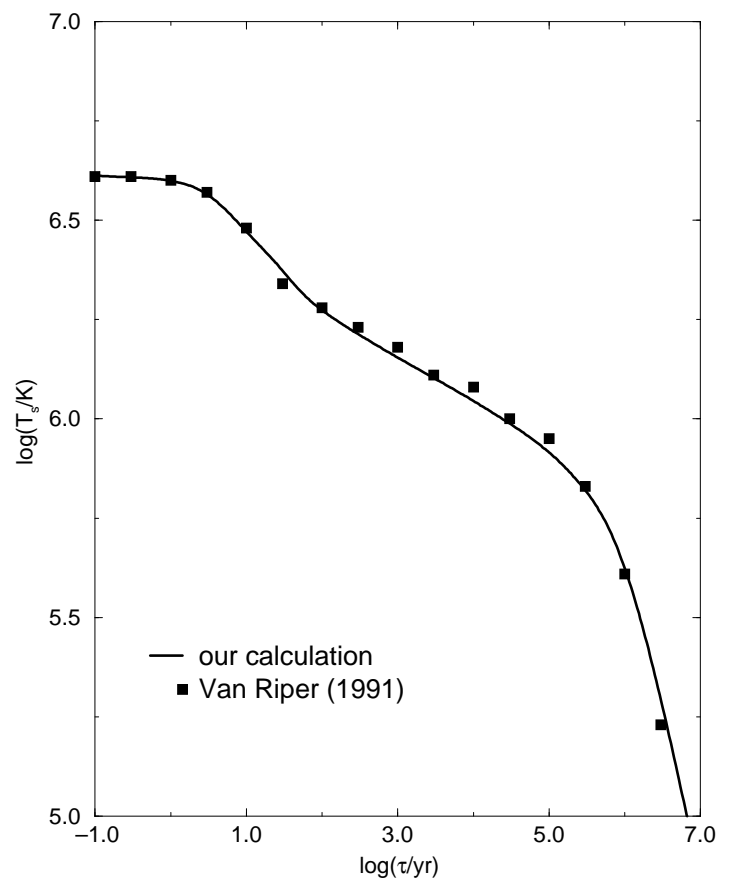

Fig. 9. Comparison of the computed surface temperature versus age with the results of Van Riper [12]. The parameters are: $M=1.4 M_{\odot}$, BPS EOS [10], no superfluidity and no enhanced cooling processes are included.

mixed phase of baryons and quarks in their interior (hybrid stars) and strange stars.

\section{$7 \quad$ Results and comparison with observed data}

\subsection{Test of the numerical code}

We tested the correctness of our numerical code by means of comparing the outcome of several cooling calculations with those obtained by other authors. As an example, in fig. 9 we compare our surface temperatures, obtained for a particular model for the equation of state, with the corresponding results obtained by Van Riper [12]. The agreement is extremely good for all star ages. To achieve this agreement, however, we had to reduce the core's thermal conductivity, which was not specified by Van Riper, from the value given in [17] by a factor of 40 . Otherwise deviations of about $\sim 20 \%$ were obtained at star ages between $\tau \sim 1$ to 10 years. The perfect agreement at later stages however is not influenced by this. 
Table 6

Survey of observed pulsar ages and luminosities, denoted $\tau$ and $L$, respectively

\begin{tabular}{|c|c|c|c|c|}
\hline Pulsar & Name & $\log \tau[\mathrm{yr}]$ & $\log L[\mathrm{erg} / \mathrm{s}]$ & Reference \\
\hline \multicolumn{5}{|c|}{ not enough data available for spectral analysis } \\
\hline $1706-44$ & & 4.25 & $32.8 \pm 0.7$ & 83 \\
\hline $1823-13$ & & 4.50 & $33.2 \pm 0.6$ & 84 \\
\hline $2334+61$ & & 4.61 & $33.1 \pm 0.4$ & 85 \\
\hline \multicolumn{5}{|c|}{ power-law-type spectra or spectra with only a high temperature componer } \\
\hline $0531+21$ & Crab & 3.09 & $35.5 \pm 0.3$ & 86 \\
\hline $1509-58$ & SNR MSH 15-52 & 3.19 & $33.6 \pm 0.4$ & 87,88 \\
\hline 0540-69 & & 3.22 & $36.2 \pm 0.2$ & 89 \\
\hline $1951+32$ & SNR CTB 80 & 5.02 & $33.8 \pm 0.5$ & 90 \\
\hline $1929+10$ & & 6.49 & $28.9 \pm 0.5$ & 4,91 \\
\hline $0950+08$ & & 7.24 & $29.6 \pm 1.0$ & 92 \\
\hline J0437-47 & & 8.88 & $30.6 \pm 0.4$ & 93 \\
\hline
\end{tabular}

spectrum dominated by a soft component

$\begin{array}{ccccc}0833-45 & \text { Vela } & 4.05 & 32.9 \pm 0.2 & 99 \\ 0656+14 & & 5.04 & 32.6 \pm 0.3 & 95 \\ 0630+18 & \text { Geminga } & 5.51 & 31.8 \pm 0.4 & 96 \\ 1055-52 & & 5.73 & 33.0 \pm 0.6 & 97\end{array}$

The age is determined by the spin down of the pulsar, the luminosities by spectral fits or the totally detected photon flux, depending on the categories (i) to (iii) listed in the text.

\subsection{Body of observed cooling data}

From the X-ray observatories Einstein, EXOSAT, and ROSAT the X-ray spectra of at least 14 pulsars have been observed to date. Unfortunately, the data are not always sufficient to extract the effective surface temperature of the corresponding pulsar. One can classify the spectra into three categories [4] (compare with table 6):

(i) The photon yields of three pulsars (PSR's 1706-44, 1823-13, and 2334+61) contain too few photons in order to perform spectral fits. The luminosities 
are calculated by using the totally detected photon flux. These pulsars are marked with triangles in the figures.

(ii) The spectra of seven pulsars, including the crab pulsar (PSR 0531+21), can only be fitted by a power-law type spectrum or a blackbody spectrum with a very high effective temperature, and an effective area much smaller than the neutron star surface. Their X-ray emission is predominated by magnetospheric emission. Therefore, their temperatures, determined from the spectral fits, are probably too high. Theses pulsars are marked with solid dots.

(iii) Finally, there are four pulsars (i.e., 0833-45 (Vela), 0656+14, 0630+18 (Ge-minga), and 1055-52) that allow two-component spectral fits, where the softer blackbody components corresponds to the actual surface emission of the neutron star and the harder to some magnetospheric emission. These pulsars are marked with squares.

When discussing spectra of neutron stars one should keep in mind that neutron stars are not black bodies, because hydrogen and helium in their atmospheres modify the blackbody spectrum. Besides, strong magnetic fields can also affect the surface emission. With respect to the luminosity determinations we note that the luminosities determined via hydrogen- or helium-atmospheric models are generally lower by a factor of about three than those determined via blackbody spectral fits. The inverse is true for the temperature which is smaller by a factor of about two. An uncertainty in temperature by a factor of two varies the luminosity, according to the Stefan-Boltzmann law $\left(L \propto T^{4}\right)$, by a factor of 16 . It is thus not appropriate to perform a comparison with surface temperatures of stars [4]. Instead one should be using the luminosities, as is the case here.

\subsection{Standard cooling calculations}

Figure 10 shows the cooling history of a neutron star with $M=1.4 M_{\odot}$ that cools down via the standard scenario only, i.e., fast cooling processes are not allowed. The different curves correspond to different models for the equation of state. One sees that the standard cooling behavior depends only weakly on the equation of state. The $\mathrm{UV}_{14}+\mathrm{UVII}$ model cools somewhat faster at later times than the other two models. This is due to the rather soft behavior of the $\mathrm{UV}_{14}+\mathrm{UVII}$ equation of state at intermediate nuclear densities (fig. 11), which leads to neutron stars with small radii (figs. 2-4). Obviously, most of the observed data are not in agreement with the assumption of standard cooling. Specifically Vela's (0833-45) luminosity lies considerably below the computed curves. The data of PSR 0630+18 (Geminga) and a few others are 


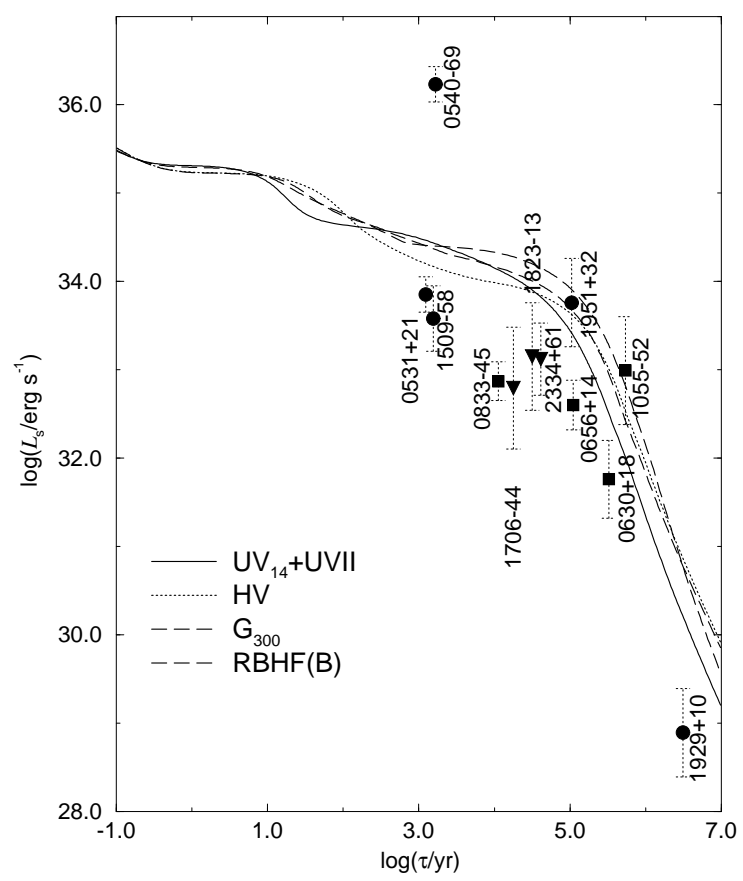

Fig. 10. Standard cooling behavior of a neutron star with mass $M=1.4 M_{\odot}$ for several different models for the EOS.

in relatively good agreement, however. ${ }^{2}$

\subsection{Enhanced cooling via direct Urca process}

As mentioned above, one way by means of which neutron stars can cool very efficiently is the direct Urca process 98 100]. This is demonstrated graphically in figs. 1113 for the equations of state $\mathrm{UV}_{14}+\mathrm{UVII}, \mathrm{HV}$, and $\mathrm{G}_{300}$. The proton fraction in the stars with $M=1.0 M_{\odot}$ is for all three cases below the threshold density for the direct Urca process. Hence these stars cool via the standard mechanism, the modified Urca process. The cooling behavior of all other stars at early stages is dominated by the direct Urca process. The sudden drop in luminosity at ages of several hundred years has its origin in the fact that the core cools down much faster through the enhanced cooling process than the crust. This causes a temperature inversion in young stars. After several hundred years, depending on the thickness of the crust, the "cooling wave", built up by the temperature gradient, reaches the star's surface which leads to the sudden luminosity drops. (An estimate of the time needed by the cooling

$\overline{2}$ Note that there is a considerable uncertainty in the ages of pulsars that are derived from the braking index, $n$, which, due to lack of information, usually is taken to be constant during a pulsar's spin-down. Moreover a canonical value of $n=3$ [13], which corresponds to a slow-down by emission of pure magnetic dipole radiation, is assumed. This too constitutes a rather crude approximation. 


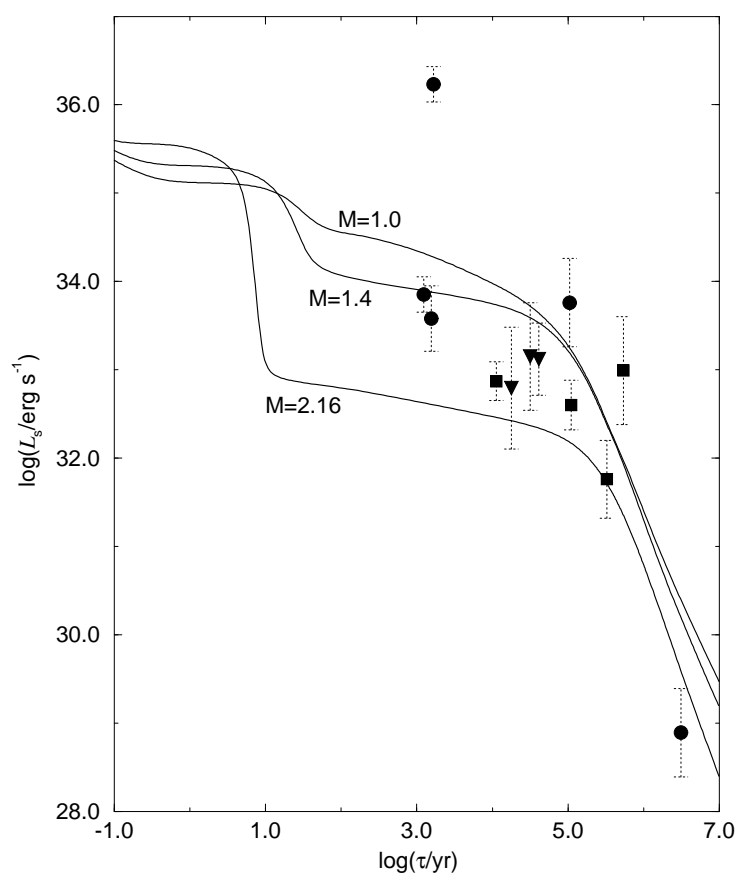

Fig. 11. Enhanced cooling of neutron stars via direct Urca process. The underlying EOS is $\mathrm{UV}_{14}+\mathrm{UVII}$. The observed data are labeled in fig. 10.

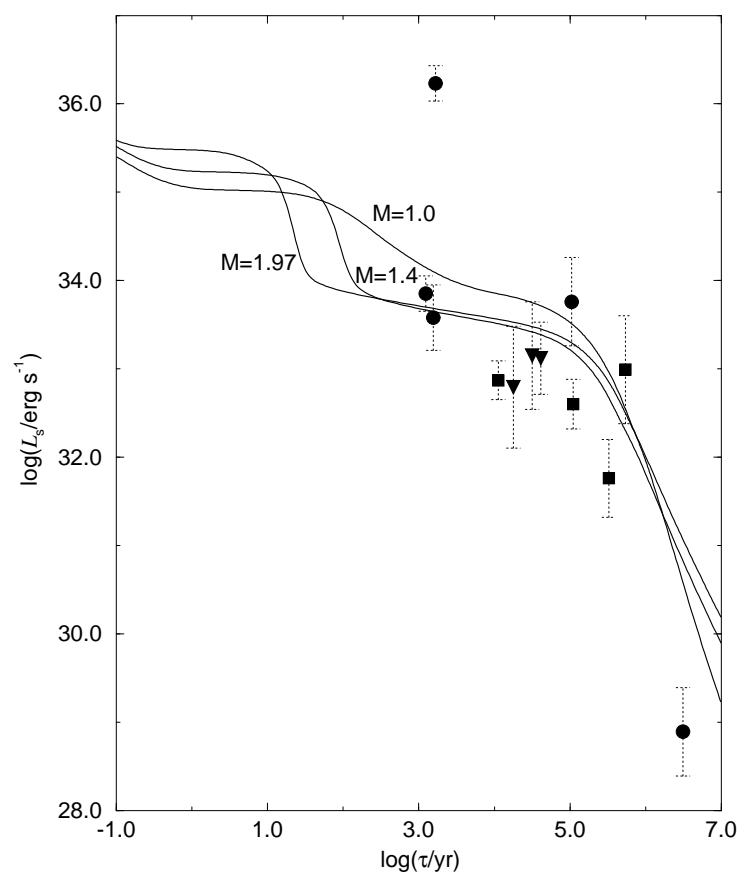

Fig. 12. Same as figure 11 but for HV. The observed data are labeled in fig. 10.

wave to reach the surface can be found in [101].) The drop of luminosity increases with massive, since the region inside the core of a star where the direct Urca process is possible grows with density. The drop is most strongly pronounced for $\mathrm{UV}_{14}+\mathrm{UVII}$. This has its origin in the ${ }^{3} \mathrm{P}_{2}$ neutron-superfluid 


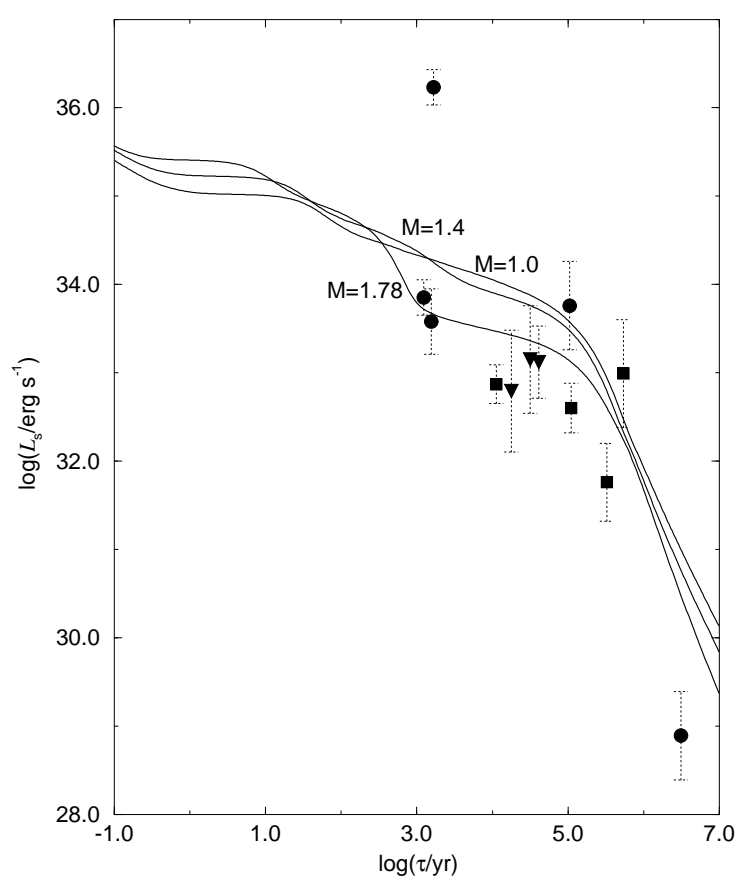

Fig. 13. Same as fig. 11 but for $\mathrm{G}_{300}$. The observed data are labeled in figure 10 . which does not extent into the central core.

Changes in the hyperon couplings strengths in matter influence the cooling curves only very little, as can be seen in fig. 14 for neutron stars of several different masses. (Note that this may be drastically different if the direct Urca process among hyperons occurs is dense stellar matter [75,76].) The star with $M=0.7 M_{\odot}$ does not contain hyperons, since its core is not dense enough to reach the threshold density for hyperon population. Hence there is only one cooling curve in this case. The situation is different for the heavier stars shown in this figure, for which the hyperon concentration increases with star mass 105. The hyperons are coupled to the meson fields either with the same strengths as the nucleons, i.e., $x_{\sigma}=x_{\omega}=x_{\rho}=1$ (cf. Eq. (27)), or are chosen such that mutual compatibility between the $\Lambda$ binding energy in saturated nuclear matter, neutron-star masses, and experimental data on hypernuclear levels is achieved (cf. sect. 3.3). As for the other figures of this section, the direct Urca process among the nucleons is included, and no superfluidity is taken into account.

\subsection{Enhanced cooling via pion condensation}

The impact of pion condensation on the cooling of neutron stars [100] is demonstrated in fig. 15. comparison with fig. 13 shows that the cooling behavior of such stars is qualitatively similar to the one of stars which cool via the direct Urca process. The threshold density of the pion condensate lies at 


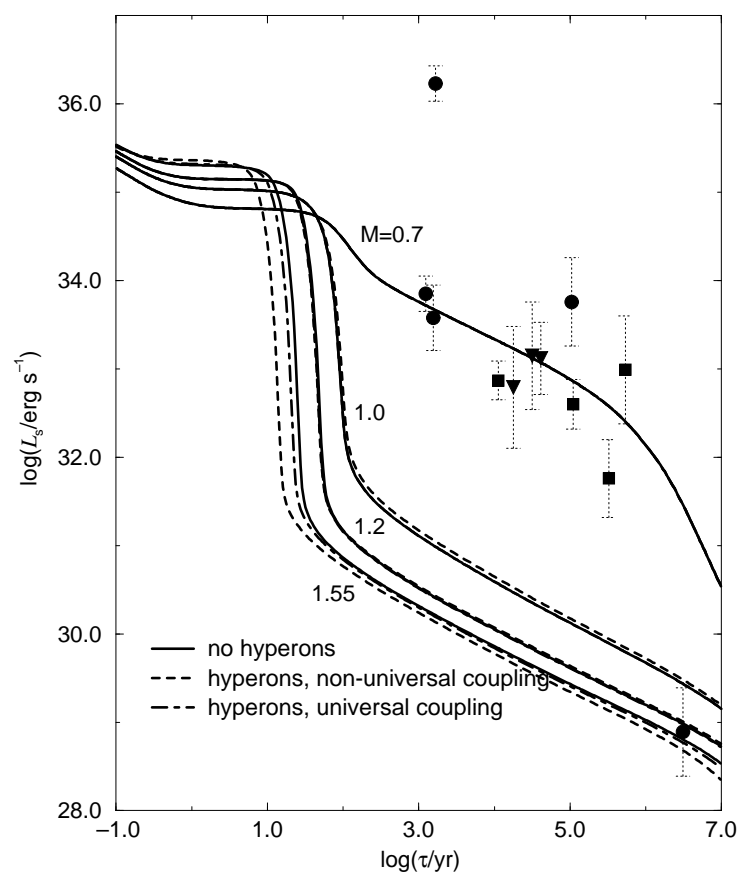

Fig. 14. Enhanced cooling of neutron stars due to direct Urca among nucleons, but for varying hyperon coupling strengths (see text). The underlying EOS is $\mathrm{G}_{\mathrm{M} 78}^{\mathrm{K} 240}$. The observed data are labeled in fig. 10.

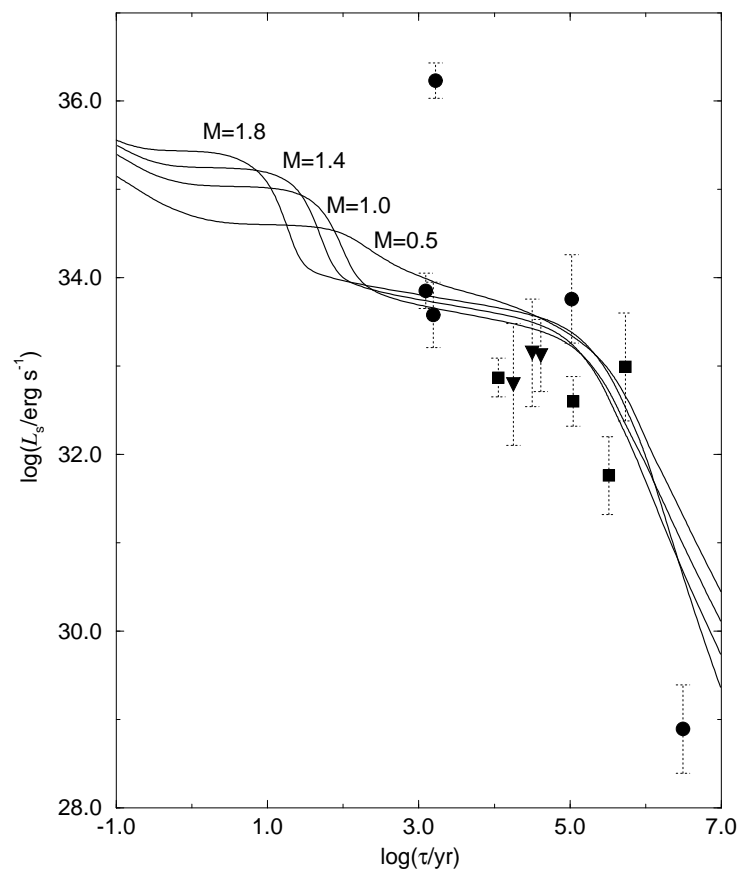

Fig. 15. Enhanced cooling of neutron stars due to pion condensation. The underlying EOS is $\mathrm{G}_{300}^{\pi}$. The observed data are labeled in fig. 10. 


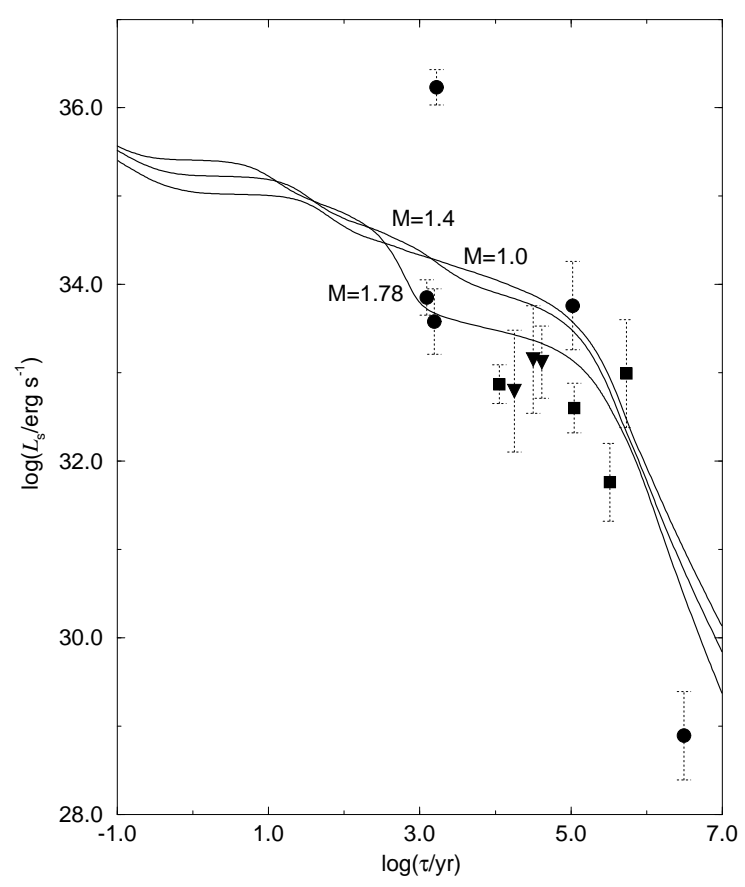

Fig. 16. Enhanced cooling of neutron stars due to kaon condensation. The underlying EOS is $\mathrm{G}_{300}$. The observed data are labeled in fig. 10 .

$3.6 \times 10^{14} \mathrm{~g} \mathrm{~cm}^{-3}$, just slightly above normal nuclear matter saturation density. Hence all stars displayed in fig. 15, with the exception of $M=0.5 M_{\odot}$, possess a pion condensate and therefore show enhanced cooling triggered by the condensate.

\subsection{Enhanced cooling via kaon condensation}

The cooling behavior of kaon-condensed stars (see also [53, 100, 102 104) is qualitatively similar to those of pion-condensed stars. This is graphically illustrated in fig. 16 for the $\mathrm{G}_{300}$ equation of state. There is however a significant quantitative difference between both types of condensation which concerns the threshold densities. It amounts for kaon condensation about $4.8 n_{0}$ [61], which is by a factor of $\sim 2.5$ larger than the threshold density established for pion condensation, $\sim 1.3 n_{0}$ [105]. A density of $4.8 n_{0}$ is reached only in the most massive star model, $M=1.78 M_{\odot}$, constructed for $\mathrm{G}_{300}$. Hence the other two models cool via the modified Urca process only. For the purpose of comparison, the central density of the $M=1.4 M_{\odot}$ neutron star amounts $2.4 n_{0}$ which lies almost by a factor of two below the kaon threshold. The situation would be different for softer models for the equation of state, which lead to higher central neutron star densities. For the broad collection of modern equations of state of ref. [25] we find central densities in $M \sim 1.4 M_{\odot}$ stars that range from about $2.3 n_{0}$ to $4.2 n_{0}$. The latter value comes much closer to the critical value 


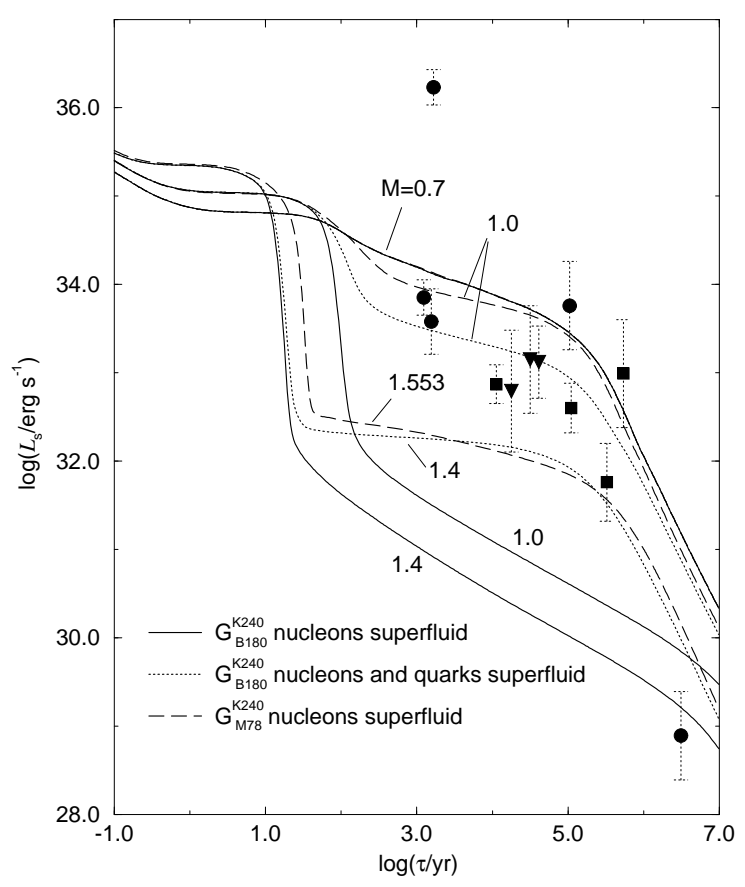

Fig. 17. Enhanced cooling of neutron stars due to an admixture of quark matter in their cores $\left(\mathrm{G}_{\mathrm{B} 180}^{\mathrm{K} 240} \mathrm{EOS}\right)$. Curves corresponding to the $\mathrm{G}_{\mathrm{M} 78}^{\mathrm{K} 240}$ EOS are without quarks. Enhanced cooling via direct Urca is taken into account too. The observed data are labeled in fig. 10.

for kaon condensation derived in [61], which, of course, is model dependent too and thus not accurately known. Clearly, the smaller the threshold density the faster the cooling of compact stars via kaon condensation will be.

\subsection{Enhanced cooling due to the presence of quark matter in the cores of neutron stars}

As discussed before, modern investigations of the possible transition of baryon matter into quark matter predict transition densities less than twice normal nuclear matter density. Such densities are easily reached in neutron stars with masses $M \approx 1.4 M_{\odot}$ [25] (see also discussion at the end of section 7.6). To simulate the cooling history of such (hybrid) stars we apply Glendenning's $\mathrm{G}_{\mathrm{B} 180}^{\mathrm{K} 240}$ equation of state, whose specific properties have been discussed in connection with table 3. The weak reaction processes of the quarks are those given in Equations (39) and (40). They speed up the energy loss of a star considerably, as is illustrated in fig. 17, where the solid curves correspond to enhanced cooling caused by the quarks (see also [12]). The dashed curves refer to stars with no quarks in their cores, which therefore cool more slowly. Note that the density in the light stars with $M<1 M_{\odot}$ is not high enough to reach the transition into quark matter. Enhanced quark cooling is therefore not possible for these stars. Finally, if the quarks should form a superfluid, 


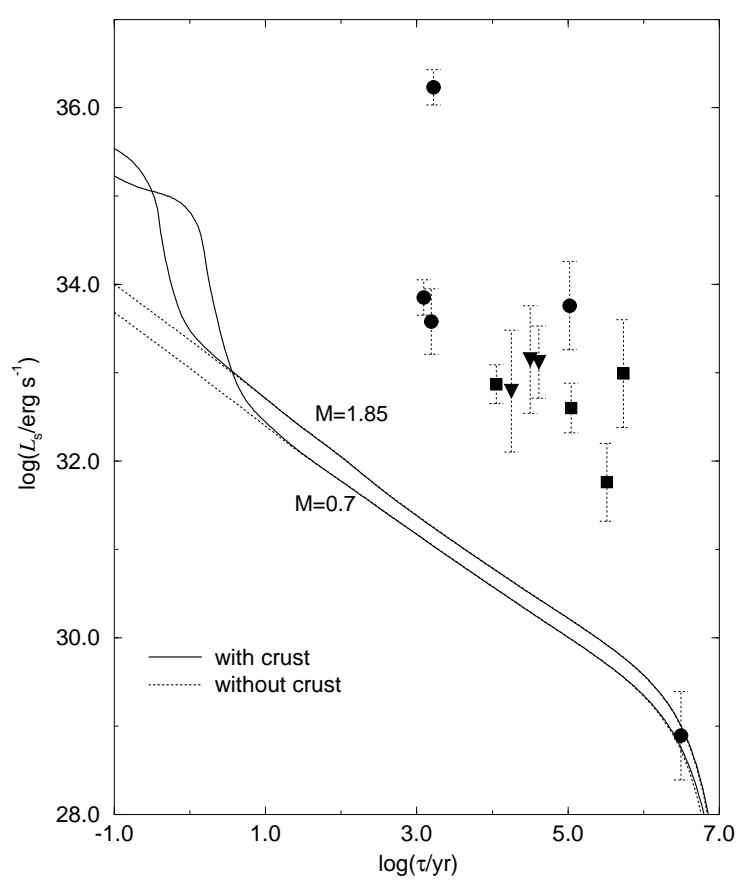

Fig. 18. Cooling behavior of strange quark matter stars of masses $M=0.7 M_{\odot}$ and $M=1.85 M_{\odot}$ with and without crust. The quarks are treated as non-superfluid. The observed data are labeled in fig. 10.

the emission of neutrinos is suppressed and cooling proceeds at a much slower pace (dotted curves). Bailin and Love have stated that quarks might become superfluid [52]. Unfortunately, there exists - as far as we know - no estimation of the gap energy of superfluid quark matter. Therefore, in order to simulated this effect, we simply assumed a plausible value of $\Delta_{\mathrm{sf}}=0.1 \mathrm{MeV}$ for the ${ }^{1} \mathrm{~S}_{0}$ pairing state of quarks, which was taken to be constant over the whole density range.

\subsection{Strange quark matter stars}

We restrict ourself to determine the cooling behavior of strange stars which are either bare or possess the densest possible hadronic crust that can be carried by such objects (inner crust density equal to neutron drip density). Figure 18 reveals that, no matter how thick the crust, such stars cool very rapidly 106. Among the body of observed luminosities, there is presently one data point that would be consistent with the assumption that the underlying object is a strange star. This interpretation is not stringent of course, because some of the enhanced cooling mechanisms studied above might do as well. If one treats the quarks as superfluid, neutrino production ceases and therefore cooling is considerably slowed down. To simulate this effect qualitatively we assumed a density-independent energy gap of $\Delta_{\text {sf }}=0.1 \mathrm{MeV}$. The numerical outcome

of such a simulation is exhibited in fig. 19. One sees that in this case the 


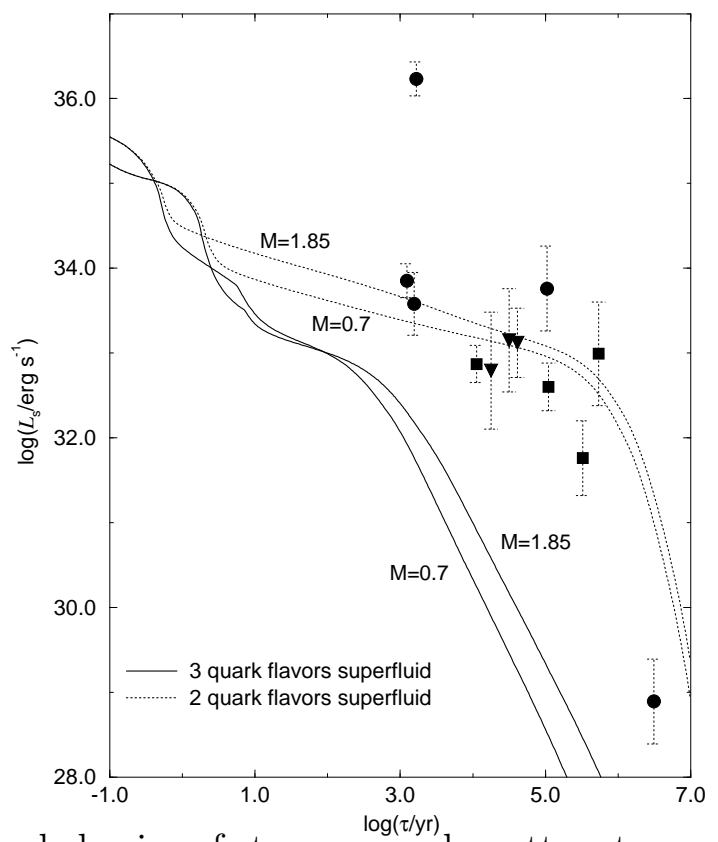

Fig. 19. Cooling behavior of strange quark matter stars with crust. The masses are $M=0.7 M_{\odot}$ and $M=1.85 M_{\odot}$. The quarks are treated as superfluid particles. The heat capacity is reduced by an exponential factor in one model, while in the other model it is kept unchanged. The observed data are labeled in fig. 10.

cooling behavior of strange stars is similar to the enhanced cooling behavior of neutron stars (compare figs. 11-17). Hence, with the exception of PSR 054069 practically all observed pulsar luminosities can now be reproduced too. A quantitative clarification of the question as to whether or not quark matter indeed becomes superfluid, and if so, at which densities, attains particular interest from astrophysics too. There is also the possibility that the quark flavors become superfluid at different densities. Consequently the heat capacity would be somewhat smaller. To simulate this possibility we reduce the heat capacity by an (arbitrary) factor of three. The consequences for the cooling behavior are shown by the dotted curves in 19 .

In closing this section, we mention that one of the main differences between the cooling behavior of neutron stars and strange quark matter stars concerns the time needed for the cooling wave to reach the surface. This time is much shorter for strange quark matter stars because their crusts have a thickness of only $\sim 0.2 \mathrm{~km}$ as opposed to $\sim 2 \mathrm{~km}$ for stars with $M \sim 1.4 M_{\odot}$.

\section{Conclusions}

We have already pointed out in section 7.2 that the observed cooling data of pulsars marked with squares in our figures appear to be the most significant ones. Primarily these data should be reproduced by theoretical cooling calculations. As we have seen above (section 7.3) the luminosities of a few pulsars, 


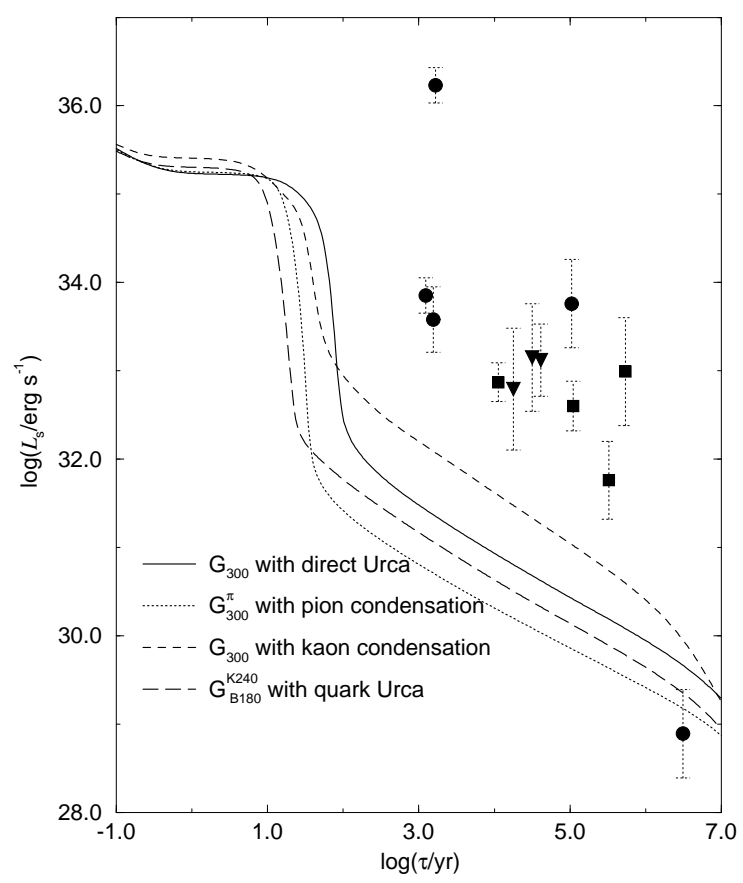

Fig. 20. Influence of several different enhanced neutrino-emission processes on the cooling of neutron stars with masses $M=1.4 M_{\odot}$, except for the kaon-condensed model where the mass is $M=1.78 M_{\odot}$ (The $1.4 M_{\odot}$ models do not possess large enough central densities to overcome the threshold density for kaon condensation). The observed data are labeled in fig. 10.

like 1951+32 and 1055-52, appear to be compatible with the standard cooling scenarios. Depending on the model employed for the equation of state, this may also be the case for Geminga and pulsars 0656+14, 2334+61 (cf. fig. 10). Others, however, like the famous Vela pulsar (PSR 0833-45) and PSR's 1706$44,1929+10$, which have rather low observed luminosities, may require the introduction of so-called fast (or enhanced) cooling processes, which are characterized by higher neutrino emission rates by means of which stars cool more rapidly. A final decision on this issue - standard versus enhanced cooling [4] is presently hampered by the uncertainties in a number of quantities, including the modified Urca neutrino-emission rate, superfluid gaps, equation of state, and mass and age of the star. Nevertheless, subject to the enormous progress that is being made presently in exploring the behavior of superdense matter [1], one may feel confident that this will change in the foreseeable future.

Figure 20 compares the influence of different enhanced neutrino emission processes, i.e., direct Urca, pion and kanon condensation, and quark Urca, on the cooling behavior of a neutron star with mass $M=1.4 M_{\odot}$. The nucleons and quarks are treated as non-superflid particles. One sees that the inclusion of any of these enhanced cooling processes reduces the star temperature too quickly in order to get agreement with the observed data points. The only exception is PSR $1929+10$. The most significant data points (squares), however, 
lie considerably above the enhanced cooling curves.

The enhanced cooling scenarios can be slowed down if one assumes that the neutrons in the cores of neutron stars are superfluid, as was the case in figs. 11-17. The only problem left then concerns the horizontal "plateaus" in these figures at star ages between $10^{2}$ and $10^{5}$ years which tend to lie somewhat too high for stars with canonical mass, $M=1.4 M_{\odot}$, to be consistent with the data. Note, however, that one gets agreement with some of the data points if the star mass is assumed to be different from the canonical value. Another possibility to get agreement consists in varying the gap energy, $\Delta_{\text {sf }}$, which, as mentioned in section 5, is not very accurately know. This is particularly the case for the high-density ${ }^{3} \mathrm{P}_{2}$ superfluid. A reduction of this gap by a factor of two, for instance, moves the cooling curves up right into the region where the oberved data is concentrated, as can be seen in fig. 21.

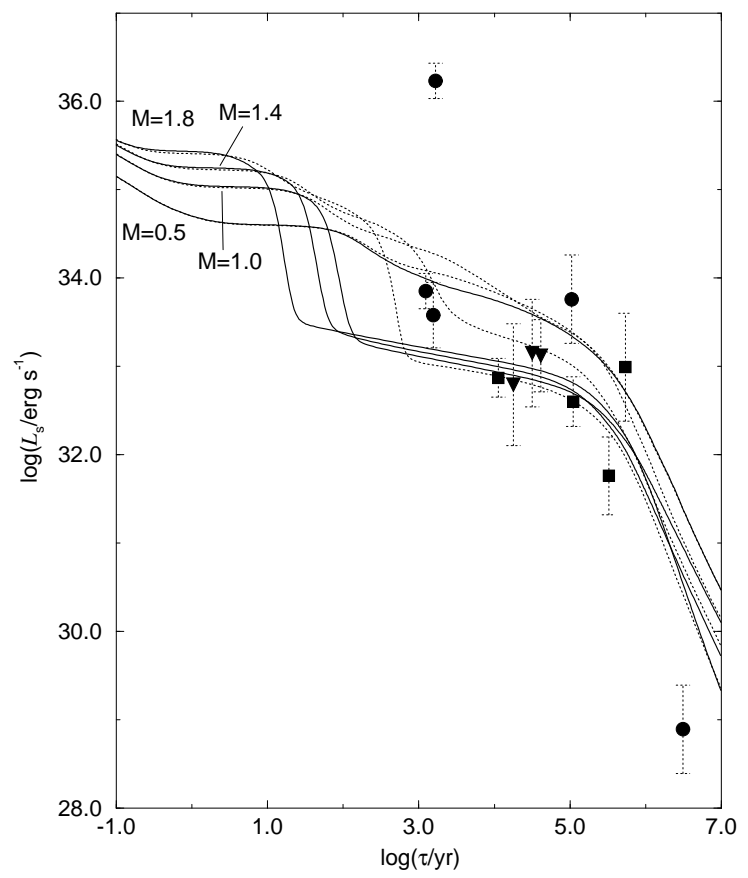

Fig. 21. Influence of changes in the superfluid ${ }^{3} \mathrm{P}_{2}$ gap on the cooling of neutron stars constructed for $\mathrm{G}_{300}$. The enhanced cooling processes are direct Urca in pion (solid curves) and kaon condensed (dotted curves) matter. The observed data are labeled in fig. 10.

Of course there are other possibilities, besides reducing the gap energy, by means of which agreement with the observed data may be achieved. For example, the superfluid phase of neutrons might not reach the center of very massive neutron stars, as is the case for stars constructed for $\mathrm{UV}_{14}+$ UVII (see fig. 11). This is due to the smaller proton fraction and the resulting higher Fermi momenta of neutrons. Another possibility is an additional cooling process not suppressed by superfluidity, as, for example, the superfluid pair-breaking pro- 
cess [71,73], or so-called internal heating of neutron stars 107 111.

\section{Summary}

We have applied a broad collection of modern, field theoretical models for the equation of state of superdense matter to the investigation of the cooling behavior of compact stars, which comprise neutron stars and their strange counterparts, the hypothetical strange stars. The models for the equation of state were derived elsewhere, using many-body approximation techniques at different levels of sophistication and complexity. Furthermore, uncertainties in the behavior of matter at extreme densities, like the phenomenon of meson condensation, superfluidity, hyperon degrees of freedom, transition to quark matter, and absolute stability of strange quark matter are incorporated in this collection.

Besides standard cooling, which, subject to uncertainties in the neutrino emissionrate of the modified Urca process, superfluid gaps etc. (cf. section 8), gives agreement between the theoretical cooling curves and the observed data for some but not all pulsars (the obtained luminosities are considerably too high in some cases), we studied various enhanced cooling mechanisms too. These are connected to higher neutrino-emission rates coming from the direct Urca process, $\pi$ - and $K$-meson condensates, and up, down and strange quarks in the cores of neutron stars. It turns out that the enhanced processes altogether cool the star models too rapidly in order to get agreement with the data. So one is left with introducing processes which delay somewhat the cooling as driven by the enhanced processes. A plausible candidate is superfluidity, of which there occur probably two different types in the cores of neutron stars, namely ${ }^{1} \mathrm{~S}_{0}$ and ${ }^{3} \mathrm{P}_{2}$. Due to the complicated theoretical analysis, the density dependences and widths of the associated superfluid gaps cannot be determined exactly, especially in the latter case. By using the more reliable ${ }^{1} \mathrm{~S}_{0}$ gaps we find that already small changes of the theoretical value for the ${ }^{3} \mathrm{P}_{2}$ gap shift the cooling curves into the region of observed data, giving an overall good agreement. An accurate determination of the superfluid gaps in neutron star matter seems therefore of particular interest.

Most interestingly, we find that-for a given star mass-not a single model for the equation of state of our collection is able to explain more than just a few data points! This makes one wonder if the observed data are possibly associated with stars of different masses. We recall that the canonical neutron star mass is about $1.4 M_{\odot}$, and the observed data 112 seem to concentrate about this value. Nevertheless, the existence of pulsars with quite different masses, e.g., neutron stars in binary systems that accrete mass from their companions, seems equally plausible. If one assumes that the observed luminosities are 
indeed coming from stars with different masses, those of Vela and Crab could amount $\sim 1 M_{\odot}$ and $\sim 1.4 M_{\odot}$, respectively, then one may have a reason for the different cooling behaviors.

As mentioned repeatedly, the absolute stability of strange quark matter would have implications of greatest importance for various branches of physics 45. It is agreed on that compact stars form ideal environments where such matter could be found. Adopting a simple model for the EOS of strange matter, we studied the cooling history of stars made up of such matter, too. If the emissivities and heat capacities of quark matter used here constitute reliable estimates, which, at present, is an unresolved issue, we find that pulsars like Geminga, Monogem, and PSR 1055-52 could be ruled out as strange pulsar candidates. There is however one pulsar, PSR 1929+10, which could be interpreted as a strange star. Of course, the QCD related uncertainties in the properties of strange matter do not allow to draw any stringent conclusions yet. In order to provide a feeling for how sensitive the cooling history of strange stars depends on the transport properties of strange matter, we also performed cooling simulations where the emissivity and heat capacity were slightly varied, due to a possible superfluid behavior of the quarks. As it turns out, this moves the cooling curves right into the region of the luminosity-age plane where most of the observed data are concentrated. So there is clearly a big need for reliably determined transport properties of strange quark matter. At the present level of development it seems to us that no definitive decision can be made as to whether the observed luminosities are being emitted from conventional neutron stars or their strange counterparts.

In summary, we find that really stringent conclusions about the behavior of superdense matter on the basis of the observed cooling data cannot be made at present. However this situation would change if besides the luminosity and the age also the mass of just a single pulsar could be determined! The rapid discovery pace of newly observed pulsars in combination with the enormous progress that has been made in observational X-ray astronomy are very encouraging in this respect.

\section{Acknowledgement:}

We would like to thank K. A. Van Riper for sending us his tables of the mantle temperature as a function of luminosity, $T_{m}\left(L_{m}\right)$, which were used in our cooling calculations. Furthermore we have benefited from conversations with Dany Page and Armen Sedrakian. This work was supported by the Director, 
Office of Energy Research, Office of High Energy and Nuclear Physics, Division of Nuclear Physics, of the U.S. Department of Energy under Contract DEAC03-76SF00098. 


\section{References}

[1] W. Greiner, H. Stöcker and A. Gallmann (eds.), Hot and Dense Nuclear Matter, NATO ASI Series B: Physics, Vol. 335, Plenum Press, New York, London (1994).

[2] J. Trümper, Royal Astronomical Society, Quaterly Journal 33 (1992) 165.

[3] J. Trümper, Science 260 (1993) 1769.

[4] H. Ögelman, in M. A. Alpar, Ü. Kiziloglu and J. van Paradijs (eds.), The Lives of the Neutron Stars, Kluwer, Dordrecht (1995), p. 101.

[5] C. W. Misner, K. S. Thorne and J. A. Wheeler, Gravitation, W. H. Freeman and Company, New York (1973).

[6] K. Thorne, ApJ 212 (1977) 825.

[7] E. Gudmundson, C. Pethick and R. Epstein, ApJ 272 (1983) 286.

[8] K. A. Van Riper, ApJ 329 (1988) 339.

[9] A. Burrows and J. M. Lattimer, ApJ 307 (1986) 178.

[10] G. Baym, C. Pethick and P. Sutherland, ApJ 170 (1971) 299.

[11] J. Negele and D. Vautherin, Nucl. Phys. A 207 (1973) 298.

[12] K. A. Van Riper, ApJ Suppl. 75 (1991) 449.

[13] S. L. Shapiro and S. A. Teukolsky, Black Holes, White Dwarfs and Neutron Stars, John Wiley \& Sons (1983).

[14] N. Itoh, Y. Kohyama, N. Matsumoto and M. Seki, ApJ 285 (1984) 758.

[15] N. Itoh, ApJ 273 (1983) 774.

[16] S. Mitake, S. Ichimaru and N. Itoh, ApJ 277 (1984) 375.

[17] O. Gnedin and D. Yakovlelv, Nucl. Phys. A 582 (1995) 697.

[18] P. Haensel, Transport properties of strange matter, CAMK preprint 228 (1991).

[19] N. Itoh, A. Tomoo, M. Nakagawa and Y. Kohyama, ApJ 339 (1989) 354.

[20] N. Itoh and Y. Kohyama, ApJ 275 (1983) 859.

[21] C. Pethick and V. Thorsson, Phys. Rev. Lett. 72 (1994) 1964.

[22] N. K. Glendenning, Phys. Rev. D46 (1992) 1274.

[23] C. Pethick, P. Ravenhall and C. Lorenz, Nucl. Phys. A584 (1995) 675.

[24] R. Wiringa, V. Fiks and A. Fabrocini, Phys. Rev. C38 (1988) 1010. 
[25] F. Weber and N. K. Glendenning, in D. Feng, G. He and X. Li (eds.), Proceedings of the Nankai Summer School, "Astrophysics and Neutrino Physics", Tianjin, China, June 17-27, 1991, World Scientific, Singapore (1993), p. 64-183.

[26] P. Martin and J. Schwinger, Phys. Rev. 115 (1959) 1342.

[27] H. Huber, F. Weber and M. K. Weigel, Phys. Rev. C50 (1994) R1287.

[28] H. Huber, F. Weber and M. K. Weigel, Phys. Rev. C51 (1995) 1790.

[29] P. Poschenrieder and M. K. Weigel, Phys. Lett. 200B (1988) 231.

[30] P. Poschenrieder and M. K. Weigel, Phys. Rev. C38 (1988) 471.

[31] F. Weber, N. K. Glendenning and M. K. Weigel, ApJ 373 (1991) 579.

[32] F. Weber and M. K. Weigel, Nucl. Phys. A505 (1989) 779.

[33] N. K. Glendenning, Nucl. Phys. A493 (1989) 521.

[34] N. K. Glendenning, unpublished (1995).

[35] N. K. Glendenning and S. A. Moszkowski, Phys. Rev. Lett 67 (1991), 2414; N. K. Glendenning, F. Weber, and S. A. Moszkowski, Phys. Rev. C45 (1992) 844.

[36] N. K. Glendenning, Nucl. Phys. (proc. Suppl.) B24 (1991) 110.

[37] A. Bodmer, Phys. Rev. D4 (1971) 1601.

[38] E. Witten, Phys. Rev. D30 (1984) 272.

[39] F. Weber, C. Kettner, M. K. Weigel and N. K. Glendenning, in G. Vassiliadis, A. Panagiotou, S. Kumar and J. Madsen (eds.), Proceedings of the International Symposium on Strangness and Quark Matter, World Scientific, Singapore (1995), p. 308.

[40] N. K. Glendenning, C. Kettner and F. Weber, ApJ 450 (1995) 253.

[41] N. K. Glendenning, C. Kettner and F. Weber, Phys. Rev. C51 (1995) 1790.

[42] N. K. Glendenning, Mod. Phys. Lett. A5 (1990) 2197.

[43] J. Madsen and M. Olesen, Phys. Rev. D43 (1991) 1069, erratum: ibid. D44 (1991) 566.

[44] R. Caldwell and J. Friedman, Phys. Lett. 264B (1991) 143.

[45] J. Madsen and P. Haensel (eds.), Strange Quark Matter in Physics and Astrophysics, Proceedings of the International Workshop, Aarhus, Denmark, Nucl. Phys. B (Proc. Suppl.) 24B (1991).

[46] C. Alcock, E.Farhi and A. Olinto, ApJ 310 (1986) 261.

[47] E. Farhi and R. Jaffe, Phys. Rev. D30 (1984) 2379. 
[48] T. Takatsuka and R. Tamagaki, Progress of Theoretical Physics Supplement 112 (1993) 27.

[49] T. Ainsworth, J. Wambach and D. Pines, Phys. Lett. B 222 (1989) 173; J. Wambach, T. L. Ainsworth, and D. Pines, Nucl. Phys. A555 (1993) 128.

[50] L. Amundsen and E. Østgaard, Nucl. Phys. A 442 (1985) 163.

[51] J. Wambach, T. Ainsworth and D. Pines, in J. Ventura and D. Pines (eds.), Neutron Stars: Theory and Observation, Kluwer Academic Publishers, Dordrecht (Netherlands) (1991), p. 37-48.

[52] D. Bailin and A. Love, Physics Reports 107 (1984) 325.

[53] D. Page, Rev. Mex. Fis. 41 (Supl. 1) (1995) 178.

[54] P. Anderson and P. Morel, Phys. Rev. 123 (1961) 1911.

[55] P. Muzikar, J. Sauls and J. Serene, Phys. Rev. D21 (1980) 1494.

[56] O. Maxwell, ApJ 231 (1979) 201.

[57] J. Chen, J. Clark, R. Davé and V. Khodel, Nucl. Phys. A555 (1993) 59.

[58] B. Friman and O. Maxwell, ApJ 232 (1979) 541.

[59] J. M. Lattimer, C. Pethick, M. Prakash and P. Haensel, Phys. Rev. Lett. 66 (1991) 2701.

[60] O. Maxwell, G. Brown, D. Campbell, R. Dashen and J. Manassah, ApJ 216 (1977) 77.

[61] V. Thorsson, M. Prakash and J. M. Lattimer, Nucl. Phys. A572 (1994) 693.

[62] V. Thorsson, M. Prakash, T. Tatsumi and C. Pethick, Phys. Rev. D52 (1995) 3739 .

[63] R. C. Duncan, S. L. Shapiro and I. Wasserman, ApJ 267 (1983) 338.

[64] N. Itoh, Y. Kohyama, N. Matsumoto and M. Seki, ApJ 280 (1984) 787.

[65] N. Itoh, Y. Kohyama, N. Matsumoto and M. Seki, ApJ 285 (1984) 304.

[66] S. Tsuruta, Canadian Journal of Physics 44 (1966) 1863.

[67] S. Tsuruta, Phys. Rep. 56 (1979) 237.

[68] G. Glen and P. Sutherland, ApJ 239 (1980) 671.

[69] M. Richardson, K. R. H.M. Van Horn and R. Malone, ApJ 255 (1982) 624.

[70] K. Nomoto and S. Tsuruta, ApJ 312 (1987) 711.

[71] D. Voskresenskii and A. Senatorov, Zh. Eksp. Teor. Fiz. 90 (1986) 1505.

[72] D. Voskresenskii and A. Senatorov, Sov. J. Nucl. Phys. 45 (1987) 411. 
[73] C. Schaab, D. Voskresenskii, A. Sedrakian and F. Weber, "Impact of Softening of Pion Exchange Modes and Superfluid Pair Braking on Cooling of Neutron Stars“, prepint, University of Munich, March 1996.

[74] J. Boguta, Phys. Lett. 106B (1981) 255.

[75] M. Prakash and M. Prakash, ApJ 390 (1992) L77.

[76] P. Haensel and O. Yu. Gnedin, A\&A 290 (1994) 458.

[77] C. Schaab, Cooling of Neutron and Strange Quark Matter Stars, Master's thesis, Ludwig-Maximilians Universität München, unpublished (1995).

[78] D. Kaplan and A. Nelson, Phys. Lett. B 175 (1986) 57.

[79] G. Brown, K. Kubodera and M. Rho, Phys. Lett. B 192 (1987) 273.

[80] C.-H. Lee and M. Rho, in G. Vassiliadis, A. Panagiotou, S. Kumar and J. Madsen (eds.), Proceedings of the International Symposium on Strangness and Quark Matter, World Scientific, Singapore (1995), p. 283.

[81] G. Brown, K. Kubodera, D. Page and P. Pizzochero, Phys. Rev. D37 (1988) 2042.

[82] T. Muto and T. Tatsumi, Phys. Lett. B 283 (1992) 165.

[83] W. Becker, P. Predehl, J. Trümper and H. Ögelman, IAU Circular No. 5554 (1992), p. 1.

[84] J. Finley and H. Ögelman, IAU Circ. No. 5787 (1993) .

[85] W. Becker, IAU Circ. No. 5805 (1993) .

[86] W. Becker and B. Aschenbach, in M. A. Alpar, Ü. Kiziloglu and J. van Paradijs (eds.), The Lives of the Neutron Stars, Kluwer, Dordrecht (1995), p. 47.

[87] F. Seward, F. Harnden, P. Murdin and D. Clark, ApJ 267 (1983) 698.

[88] E. Trussoni, W. Brinkmann, H. Ögelman, G. Hasinger and B. Aschenbach, A\&A 234 (1990) 403.

[89] J. P. Finley, H. Ögelman, G. Hasinger and J. Truemper, ApJ 410 (1993) 323.

[90] S. Safi-Harb and H. Ögelman, in M. A. Alpar, Ü. Kiziloglu and J. van Paradijs (eds.), The Lives of the Neutron Stars, Kluwer, Dordrecht (1995), p. 53.

[91] S. Yancopoulos, T. Hamilton and D. Helfland, Bull. American Astron. Soc. 25 (1993) 912.

[92] F. Seward and Z.-R. Wang, ApJ 332 (1988) 199.

[93] W. Becker and J. Trümper, Nature 365 (1993) 528.

[94] H. Ögelman, J. Finley and H. Zimmermann, Nature 361 (1993) 136.

[95] J. P. Finley, H. Ögelman and Ü. Kiziloglu, ApJ 394 (1992) L21. 
[96] J. Halpern and M. Ruderman, ApJ 415 (1993) 286.

[97] H. Ögelman and J. Finley, ApJ 413 (1993) L31.

[98] D. Page and J. H. Applegate, ApJ Lett. 394 (1992) L17.

[99] O. Yu. Gnedin and D. G. Yakovlev, Astron. Lett. 19 (1993) 104.

[100] H. Umeda, K. Nomoto, S. Tsuruta, T. Muto, and T. Tatsumi, ApJ 431 (1994) 309; H. Umeda, S. Tsuruta, and K. Nomoto, ApJ 433 (1994) 256.

[101] J. M. Lattimer, K. A. V. Riper, M. Prakash and M. Prakash, ApJ 425 (1994) 802.

[102] D. Page and E. Baron, ApJ Lett. 354 (1990) L17.

[103] D. Page, in M. R. Strayer and M. W. Guidry (eds.), Nuclear Physics in the Universe, Adam Hilger \& Co., Bristol (1993), p. 151.

[104] D. Page, ApJ 428 (1994) 250.

[105] N. K. Glendenning, ApJ 293 (1985) 470.

[106] D. Page, in R. Huerta and M. A. Perez (eds.), High Energy Phenomonology, World Scientific, Singapore (1992), p. 347.

[107] N. Shibazaki and F. Lamb, ApJ 346 (1989) 808.

[108] H. Umeda, N. Shibazaki, S. Tsuruta and K. Nomoto, ApJ 408 (1993) 186.

[109] A. Sedrakian and D. Sedrakian, ApJ 413 (1993) 658.

[110] A. Reisenegger, ApJ 442 (1995) 749.

[111] K. Van Riper, B. Link and R. I. Epstein, ApJ 448 (1995) 294.

[112] S. Thorsett, Z. Arzoumanian, M. McKinnon and J. Taylor, ApJ 405 (1993) L29. 